\title{
Spatial predictive properties of built environment characteristics assessed by drop-and-spin virtual neighborhood auditing
}

Jesse J. Plascak ${ }^{1,2^{*}} \mathbb{D}$, Mario Schootman ${ }^{3}$, Andrew G. Rundle ${ }^{4}$, Cathleen Xing ${ }^{1}$, Adana A. M. Llanos ${ }^{1,2}$, Antoinette M. Stroup ${ }^{1,2,5}$ and Stephen J. Mooney ${ }^{6}$

\begin{abstract}
Background: Virtual neighborhood audits have been used to visually assess characteristics of the built environment for health research. Few studies have investigated spatial predictive properties of audit item responses patterns, which are important for sampling efficiency and audit item selection. We investigated the spatial properties, with a focus on predictive accuracy, of 31 individual audit items related to built environment in a major Metropolitan region of the Northeast United States.

Methods: Approximately 8000 Google Street View (GSV) scenes were assessed using the CANVAS virtual audit tool. Eleven trained raters audited the $360^{\circ}$ view of each GSV scene for 10 sidewalk-, 10 intersection-, and 11 neighborhood physical disorder-related characteristics. Nested semivariograms and regression Kriging were used to investigate the presence and influence of both large- and small-spatial scale relationships as well as the role of rater variability on audit item spatial properties (measurement error, spatial autocorrelation, prediction accuracy). Receiver Operator Curve (ROC) Area Under the Curve (AUC) based on cross-validated spatial models summarized overall predictive accuracy. Correlations between predicted audit item responses and select demographic, economic, and housing characteristics were investigated.

Results: Prediction accuracy was better within spatial models of all items accounting for both small-scale and largespatial scale variation (vs large-scale only), and further improved with additional adjustment for rater in a majority of modeled items. Spatial predictive accuracy was considered 'Excellent' $(0.8 \leq \mathrm{ROC}$ AUC $<0.9)$ for full models of all but four items. Predictive accuracy was highest and improved the most with rater adjustment for neighborhood physical disorder-related items. The largest gains in predictive accuracy comparing large- + small-scale to large-scale only models were among intersection- and sidewalk-items. Predicted responses to neighborhood physical disorder-related items correlated strongly with one another and were also strongly correlated with racial-ethnic composition, socioeconomic indicators, and residential mobility.
\end{abstract}

Conclusions: Audits of sidewalk and intersection characteristics exhibit pronounced variability, requiring more spatially dense samples than neighborhood physical disorder audits do for equivalent accuracy. Incorporating rater

*Correspondence: jesse.plascak@rutgers.edu

1 Department of Biostatistics and Epidemiology, Rutgers School of Public Health, Piscataway, NJ, USA

Full list of author information is available at the end of the article

(c) The Author(s) 2020. This article is licensed under a Creative Commons Attribution 4.0 International License, which permits use, sharing, adaptation, distribution and reproduction in any medium or format, as long as you give appropriate credit to the original author(s) and the source, provide a link to the Creative Commons licence, and indicate if changes were made. The images or other third party material in this article are included in the article's Creative Commons licence, unless indicated otherwise in a credit line to the material. If material is not included in the article's Creative Commons licence and your intended use is not permitted by statutory regulation or exceeds the permitted use, you will need to obtain permission directly from the copyright holder. To view a copy of this licence, visit http://creativeco mmons.org/licenses/by/4.0/. The Creative Commons Public Domain Dedication waiver (http://creativecommons.org/publicdomain/ zero/1.0/) applies to the data made available in this article, unless otherwise stated in a credit line to the data. 
effects into spatial models improves predictive accuracy especially among neighborhood physical disorder-related items.

Keywords: Virtual neighborhood audit, Built environment, Kriging, Spatial autocorrelation, Spatial prediction

\section{Background}

Characteristics of the built environment measured through visual observation have been associated with various health-related factors and outcomes, including physical activity [1, 2], obesity [3], injuries [4], violence [5], diabetes [6], and depression [7, 8]. For example, one experimental study of vacant lot "cleaning and greening"-removal of garbage and debris, repairing structures or improving yard conditions-reported lower resident perception of crime and fear for safety along with lower police-reported crime comparing experimental to control regions [5]. Other studies have found more walkable environments-presence of sidewalks, condition of sidewalks, density of destinations-associated with greater physical activity and less diabetes $[1,2,6]$. Besides physical activity, hypothesized pathways through which built environment factors might influence health include substance use and psychosocial stress [9]. Identifying specific built environment characteristics that might be associated with health outcomes is important for motivating place-based interventions of the built environment $[5,10,11]$. However, associations of many of these studies are small, underscoring the importance of well-designed measures of the built environment that maximize accuracy [12].

Neighborhood auditing (i.e., "systematic social observation", "systematic field observation") is a systematic method used to assess specific built environment characteristics that might influence health behaviors and outcomes [13-15]. Such audits were initially conducted by in-person observations along street segments [16], but recent and readily available residential imagery from mapping- and advertisement-based businesses such as Google (i.e., Google Street View) have led to development of virtual neighborhood audit tools and protocols $[14,17-20]$. Studies comparing in-person to virtual neighborhood audits have concluded that virtual audits are reliable, valid, time-, and resource-efficient methods for assessing visual neighborhood characteristics, especially when those characteristics are conspicuous and more stable over time (e.g., sidewalk present, pedestrian signal, building conditions, etc.) [17, 19, 21-25].

Although recognizing the potential for large-scale built environment characterization, most virtual neighborhood audit studies have assessed pre-defined and small areas surrounding the residence of participants of an extant health study [21]. Few studies randomly sampled potential audit locations with the intent of generating geographically-generalizable estimates of built environment characteristics across large areas (larger than typical U.S. counties or large cities) [26]. In line with the original, in-person practice of neighborhood audits, most virtual audit tools and protocols that have been developed to date utilize street segments surrounding the residence of study participants as the sampling unit. Segment-based audits have prevailed over time despite previous studies reporting that such commonly audited built constructs as walkability and neighborhood physical disorder-indicated by presence of garbage, graffiti, poor building and yard conditions, etc.- positively spatially autocorrelate at distances up to 1000 meters [27-30]. This spatial autocorrelation, or notion that "...pairs of observations taken nearby are more alike than those taken farther apart" [31] suggests that segment-based audits might not be the most efficient sampling unit because information across a typical segment is partially redundant. Despite the few reports of spatially autocorrelated constructs, the spatial trend, autocorrelation, and predictive performance (hereafter, 'spatial properties') of models of individual audit items has yet to be systematically and thoroughly investigated.

A recent adaptation to virtual audits has been developed called "drop-and-spin" where observations are limited to a single $360^{\circ}$ view around a virtual scene, as opposed to traversing the entire segment [32]. This method was explicitly developed to test whether a surface of estimated built environment measures across a study region could be generated based on the spatial properties of resulting audit responses. Previously reported test-retest and inter-rater reliability of drop-and-spin auditing is similar to that of segment-based audits of identical items [32]. Moreover, the median item-location rating time of "drop-and-spin" auditing (7.3 s) is similar to that of the fastest reported segment-based method (7.9 s) [20], and twice as fast as typical times (15 s) [4, 14, $17-20,25,26,33-38]$. However, it is not known whether, or to what degree, drop-and-spin audit responses exhibit spatial properties that are required for accurate spatial prediction.

Estimates of the spatial properties of neighborhood audit item response patterns are important for several reasons. First, investigation of "drop-and-spin" audit item-specific spatial properties is critical for motivating whether point-based auditing might be used in 
place of segment-based. When audit item responses demonstrate spatial autocorrelation and high spatial predictive accuracy, point-based audits of a sample of points may characterize neighborhood conditions as accurately as a segment-based census of street segments but at lower cost. Lack of any spatial components to audit responses would indicate that dropand-spin auditing is only useful at the point location assessed and cannot be generalized any further.

Second, investigation of spatial prediction performance of specific audit items, segment- or pointbased, has yet to be investigated. Such results are important as use of such spatial prediction methods to yield estimates of built environment characteristics across epidemiologic study regions have been increasingly recommended [39-42], including characteristics assessed from virtual neighborhood audit studies [25].

Third, previous audit studies of rater-reliability indicate large variability in test-retest and inter-rater agreement of audit item responses [23, 27, 32, 43]. If this disagreement is systematic as opposed to random (e.g., one rater consistently rates the same sidewalk quality as worse than another does), then it is possible to improve spatial prediction accuracy by accounting for this source of inaccuracies in measurement [44, 45].

Fourth, observable built environment features such as pedestrian amenities are indicators of historical social processes that have distinct spatial distributions [46, 47]. Individual audit item indicators of healthrelevant constructs vary at different spatial scales and understanding these variations is important to informing how best to build constructs from response patterns as well as the processes influencing these patterns. For example, number of traffic lanes and presence of sidewalks are both indicators of pedestrian-friendliness at a given location [48], but number of lanes is very street-dependent, whereas presence of sidewalks is not, and so these should not be combined into a single measure for spatial interpolation.

The purpose of this study was to investigate the spatial properties, with a focus on spatial prediction, of 31 commonly-assessed characteristics of the built environment measured using the newly developed drop-and-spin virtual neighborhood audit method. Relationships between predicted audit item responses as well as between predicted audit responses and various neighborhood characteristics were explored to inform relationships within audit item responses and between social factors and audit item responses.

\section{Methods}

Study sample

Virtual neighborhood audit locations were generated across non-highway roads within Essex County, NJ. New Jersey is the most densely populated U.S. state (1195.5 people per square mile), and Essex is the most populous county in NJ $(783,969)$ [49]. Essex County contains Newark, NJ and other densely populated urban areas to the east. Newark International airport is the area with few audit locations that is immediately southeast of the inset (Fig. 1). Numerous suburban communities and non-residential parks (i.e., indicated by no roads/audit locations) lie to the less dense western region. Details of the sampling scheme, audit training protocol, audit item prevalence, and audit item reliability have been previously described [32]. In brief, iterative GIS operations-random point generation, point-to-point near distance calculation, integration of points within a specific distance of separation, event collection, snapping to road filewere completed to generate points along non-highway roads. In order to have enough power to test for spatial autocorrelation, which studies of similar constructs have reported occur within distances of 1000 meters, the above GIS operations were repeated until the average point-to-point near distance was within 1 standard deviation of 150 meters (mean $=142$ meters, standard deviation $=18$ meters), resulting in 8262 total candidate audit locations (25.3 per square $\mathrm{km}$ ) (Fig. 1).

Eleven raters were trained during in-person sessions using a standardized protocol and manual [32]. Thirtyone audit items-10 intersection-related, 10 sidewalkrelated, and 11 neighborhood physical disorder-related, were assessed at each audit location. Raters were assigned audit locations at random throughout the study region. Groups of audit items were assigned to raters according to item similarity (i.e., intersection-related or sidewalk-related) or belonging to a theoretical construct (i.e., neighborhood physical disorder/aesthetics). Full audit item wording and response values are displayed in Table 1. The virtual neighborhood audit platform called CANVAS was used in this study Although numerous virtual auditing platforms exist (see Rzotkiewicz et al.), CANVAS is arguably the most frequently used platform and efficiently combines GSV scene visualization with audit items in a consistent and clear interface $[14,21]$. Audit items were chosen from previous scales, or theoretically related to constructs of previous scales, that assess observable built environment characteristics related to walkability, pedestrian infrastructure, and physical disorder [13, 29, 50, 51]. 


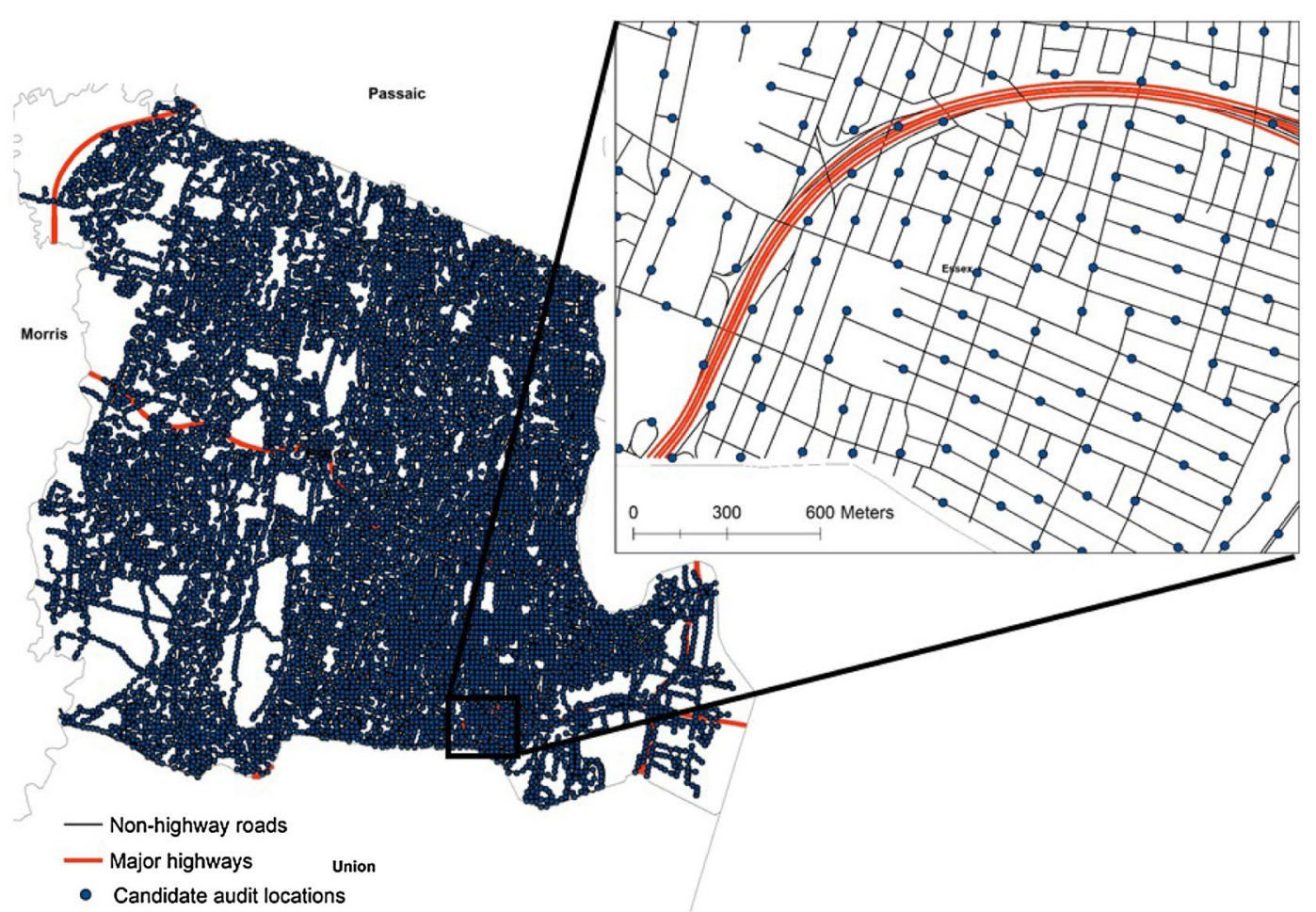

Fig. 1 8,262 candidate neighborhood audit locations assessed for 31 different built environment characteristics, Essex County, New Jersey

\section{Statistical analysis}

A workflow of all statistical analyses is displayed in Fig. 2. Nonparametric, spatially varying probability surfaces of each item response $=$ 'Yes'/'1' (Table 1 ) were created to visualize the spatial distribution of each binomially distributed item response [52]. Estimated probabilities were calculated following an isostropic, Gaussian kernel where the kernel smoothing bandwidth distance was selected via cross-validation as the distance which minimized the negative likelihood among all candidate distances [53]. Each resulting probability surface was shaded via a divergent color scheme with red and blue hue proportional to estimated probability of 'Yes'/'1' and white equal to the overall probability of each item. Thus, dark red areas are higher than average probability of 'Yes'/'1' and dark blue areas are lower than average probability of 'Yes' $/$ ' 1 '.

Item-specific analysis of spatial structure and prediction accuracy with and without statistical adjustment for rater proceeded as follows. First, we divided the data into 90-10 training-validation datasets. Using the training dataset, we then fit a logistic regression model of audit item responses, adjusting for 3rd order spatial covariates only. This detrending for large spatial scale relationships is recommended to meet the spatial statistical assumption of spatial stationarity, which in this case requires that audit item response patterns depend only on their relative positioning between one another and not their absolute positioning within the study region [31]. Next, we fit the same model of audit item responses with the addition of rater as an additional covariate (i.e., rater identity as a 4-level dummy code). Adjustment for rater will allow us to investigate whether variation in audit item responses by rater influences spatial prediction performance of audit item response patterns. Deviance residuals of each model were then assessed for spatial structure. The full model with notation for the Deviance residual was as follows: 
Table 1 Audit item name, full wording, response categorization, and prevalence

\begin{tabular}{|c|c|c|}
\hline Audit item (n/\% yes) & Question & Response categorization \\
\hline \multicolumn{3}{|l|}{ Neighborhood physical disorder-related } \\
\hline Garbage (3285/41.2) & $\begin{array}{l}\text { Is there garbage, litter, or broken glass in the } \\
\text { street or on the sidewalks ( } \geq 5 \text { items)? }\end{array}$ & Yes (1) vs no (2) \\
\hline Abandoned cars (13/0.2) & Are there abandoned cars? & Yes (1) vs no (2) \\
\hline Building conditions $\geq$ moderate (6349/86.3) & $\begin{array}{l}\text { How would you rate the condition of most of the } \\
\text { buildings? }\end{array}$ & $\begin{array}{l}\text { Very well/Moderately well kept (1) vs fair/poor } \\
\text { condition ( } 2 \text { ) }\end{array}$ \\
\hline Yard conditions $\geq$ moderate $(5388 / 87.7)$ & $\begin{array}{l}\text { How would you rate the condition of most of the } \\
\text { yards? }\end{array}$ & $\begin{array}{l}\text { Very well/Moderately well kept (1) vs fair/poor } \\
\text { condition (2) }\end{array}$ \\
\hline Dumpster (706/8.9) & Are there dumpsters visible? & $\geq 1$ (1) vs None (2) \\
\hline Graffiti (904/11.3) & $\begin{array}{l}\text { Is there graffiti, or evidence of graffiti that has } \\
\text { been painted over, on buildings, signs, or walls? }\end{array}$ & Yes (1) vs no (2) \\
\hline Boarded/burned building (502/6.3) & $\begin{array}{l}\text { Do you see boarded up, abandoned, or burned } \\
\text { out buildings? }\end{array}$ & Yes (1) vs no (2) \\
\hline Outdoor seating $(2131 / 27.1)$ & $\begin{array}{l}\text { Do you see any outdoor seating (e.g., benches, } \\
\text { porch swings, restaurant seating)? }\end{array}$ & Yes (1) vs No (2) \\
\hline Team sports $(187 / 2.4)$ & $\begin{array}{l}\text { Do you see any team sports equipment (e.g., } \\
\text { street hockey nets, soccer nets, basketball } \\
\text { hoops)? }\end{array}$ & Yes (1) vs no (2) \\
\hline Yard decorations (3932/49.9) & $\begin{array}{l}\text { Do you see any exterior or yard decorations (e.g. } \\
\text { banners/flags, seasonal decorations, water } \\
\text { fountains/statues)? }\end{array}$ & Yes (1) vs no (2) \\
\hline Fences $(6390 / 80.3)$ & $\begin{array}{l}\text { Do you see fences separating adjoining yards/ } \\
\text { properties or separating yards from the street? }\end{array}$ & Yes (1) vs no (2) \\
\hline \multicolumn{3}{|l|}{ Sidewalk-related } \\
\hline Sidewalk present (5462/68.2) & $\begin{array}{l}\text { What type of sidewalk or path (paved or } \\
\text { unpaved) is there? }\end{array}$ & Sidewalk (1) vs none (2) \\
\hline Complete sidewalk (4948/90.4) & Is the sidewalk complete/continuous? & Complete (1) vs incomplete (2) \\
\hline Sidewalk condition (3535/64.7) & $\begin{array}{l}\text { In what condition is the sidewalk or pedestrian } \\
\text { path? }\end{array}$ & Good (1) vs fair/poor (2) \\
\hline Sidewalk width (872/15.9) & How wide is the sidewalk? & $\geq 4$ feet (1) vs < 4 feet $(2)$ \\
\hline Sidewalk from curb distance $(71 / 1.3)$ & How far is the sidewalk or path from the curb? & $\geq 5$ feet (1) vs $<5$ feet ( 2 ) \\
\hline Car obstruction (340/6.2) & Do parked cars obstruct the path? & Yes (1) vs no (2) \\
\hline Garbage can obstruction (301/5.5) & Do garbage cans obstruct the path? & Yes (1) vs no (2) \\
\hline Pole or sign obstruction (262/5.8) & Do poles or signs obstruct the path? & Yes (1) vs no (2) \\
\hline Other obstruction $(445 / 8.1)$ & Does anything else obstruct the path? & Yes (1) vs no (2) \\
\hline Curb cuts $(2585 / 52.1)$ & Do you see any curb cuts? & Yes (1) vs no (2) \\
\hline \multicolumn{3}{|l|}{ Intersection-related } \\
\hline Clear intersection (4741/60.1) & Can you clearly see an intersection? & Yes (1) vs no (2) \\
\hline Pedestrian crossing sign (860/17.9) & Is there a pedestrian crossing warning sign? & Yes (1) vs no (2) \\
\hline Pedestrian signal $(718 / 15.1)$ & Is there a pedestrian signal? & Yes (1) vs no (2) \\
\hline Pedestrian crossing marks (2975/64.0) & $\begin{array}{l}\text { Consider places intended for pedestrians to cross } \\
\text { the street. Are these places marked for pedes- } \\
\text { trian crossing? }\end{array}$ & All/some (1) vs none (2) \\
\hline Type of pedestrian crosswalk marks (2076/68.4) & How is the road marked at crosswalks? & Painted solid lines (1) vs other (2) \\
\hline Traffic signal type (3438/73.8) & What kind of traffic signal can you see? & Traffic signal, stop sign, yield sign (1) vs none (2) \\
\hline One-way street $(846 / 10.7)$ & Is the street a one-way or a two-way street? & One-way (1) vs two-way (2) \\
\hline Number of lanes (1183/14.9) & How many lanes are there for cars? & Many $(2+)(1)$ vs few $(\leq 2)(2)$ \\
\hline Presence of highway (579/7.3) & $\begin{array}{l}\text { Can you see a highway (elevated or below } \\
\text { ground) here? }\end{array}$ & This is a highway (1) vs not a highway (2) \\
\hline Highway is barrier (29/19.9) & Is the highway a barrier to walking? & Cannot be overcome (1) vs can be overcome (2) \\
\hline
\end{tabular}




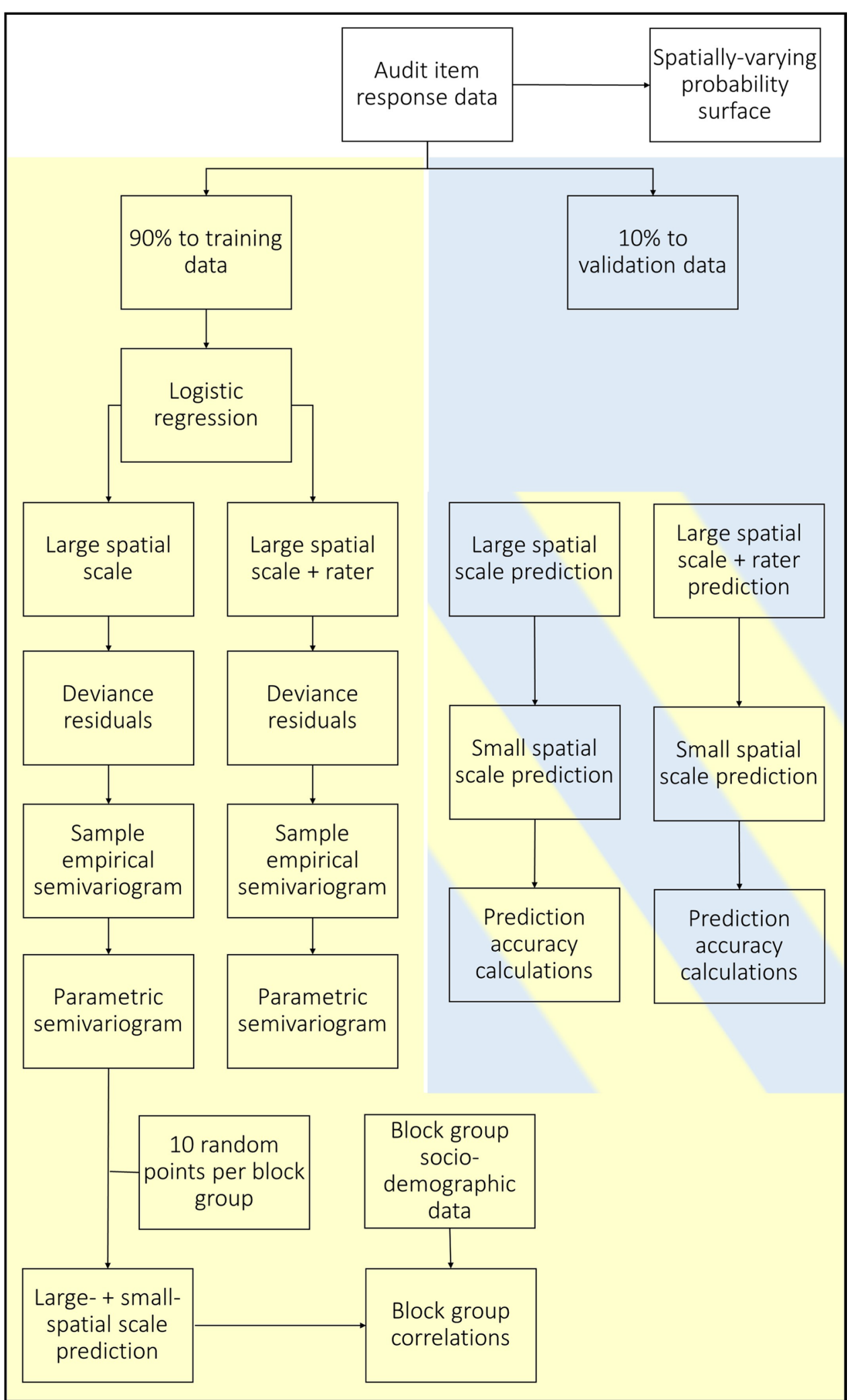

Fig. 2 Flow chart of analysis plan to investigate the spatial properties of neighborhood audit item responses 


$$
\begin{aligned}
& g(x)= \\
& \left.\begin{array}{c}
\beta_{0}+\beta_{1} \text { latitude }+\beta_{2} \text { latitude }^{2}+\beta_{3} \text { latitude }^{3} \\
+\beta_{4} \text { longitude }+\beta_{5} \text { longitude }{ }^{2}+\beta_{6} \text { longitude }{ }^{3}+\beta_{7} \text { latitude } * \text { longitude }_{\text {longtial trend }}
\end{array}\right\} \begin{array}{l}
\text { Large-scale } \\
\text { spatial }
\end{array} \\
& \left.+\beta_{8} \text { rater } 1+\beta_{9} \text { rater } 2+\beta_{10} \text { rater } 3\right\} \text { Rater variability } \\
& +\varepsilon \quad\} \text { Residual variation analyzed for } \\
& \text { small-scale spatial variability }
\end{aligned}
$$

where the function $g(\mathbf{x})$ is the logit of the probability of the audit response $=$ ' 1 '/'Yes', latitude and longitude values were centered (i.e., mean $=0$ ) in anticipation of subsequent algorithm convergence issues and to minimize polynomial estimate collinearity, rater 1-3 are dummy coded with rater 4 as the reference level, and $\varepsilon$ represents the residual which is subsequently analyzed for smallscale spatial structure. Collectively, $\beta 0-7$ describe the large-scale spatial trend, $\beta 8-10$ rater variability, and $\varepsilon$ variability in audit item responses not explained by $3 \mathrm{rd}$ order spatial trends or raters, but which could contain spatially autocorrelated audit item responses that can be used to predict unknown residuals as a function of known residual values and the distances between known and unknown values.

Experimental semivariograms of Deviance residuals within the training dataset were calculated for each audit item, with and without adjustment for rater, using identical parameters (see Additional file 1 "Supplementary methods of spatial analyses": for details of spatial analyses). Local Ordinary Kriging (OK) was performed to predict Deviance residuals from audit locations within the validation dataset based on Deviance residuals and estimated covariance parameters from theoretical semivariograms of the validation dataset. Local OK, as opposed to global OK, was performed for practical reasons; Kriging based on all points within the validation dataset was computationally prohibitive and likely statistically unnecessary given the estimated ranges that were oftentimes far shorter than $13.2 \mathrm{~km}$, let alone $26.4 \mathrm{~km}$ (Table 2). The local OK radius was set at $1.3 \mathrm{~km}$ (1/10th max semivariogram distance) or the minimum distance required to include 30 training points in the prediction of the Deviance value of the specific validation location, as recommended [54]. Others have used similar "Regression Kriging" or "Kriging with External Drift" methods based on manual detrending $[44,45,55,56]$.

The assumption of spatial isotropy, or invariance of spatial structure as a function of direction between locations, was assessed for each set of Deviance residuals by fitting directional-specific experimental and theoretical semivariograms via the same procedures and parameters used to fit omnidirectional semivariograms. Eight separate directions, uniformly dividing the unit circle $\left(0^{\circ} / 180^{\circ}, 22.5^{\circ} / 202.5^{\circ}, 45^{\circ} / 225^{\circ}\right.$, etc. $)$, were specified and anisotropy assessed visually based on experimental and theoretical semivariograms. Qualitative assessments of anisotropy violations were made-'None', ' $\geq$ Mid-range', 'Yes'-based on a combination of semivariogram behavior about the nugget, sill, and range. Comparing within directional semivariograms as well as between directional and omnidirectional semivariograms, 'None' indicates nearly identical semivariograms, ' $\geq$ Mid-range' indicates very similar semivariogram properties within estimated ranges of the majority of semivariograms, and 'Yes' indicates evidence of anisotropy (with directional violation noted).

Prediction accuracy was measured by root mean squared prediction error (RMSPE) calculated from predicted and observed audit responses within the validation datasets of each audit item model (with and without adjustment for rater). Kriging-predicted audit item response residuals were calculated by back-transforming the Kriging-estimated Deviance residuals to raw residuals. Validation dataset trend components were calculated by the score method and using the model built from the training dataset (e.g., mean response, 3rd order spatial trend, and rater adjustment if applicable). The predicted audit item response of the validation dataset was obtained by summing the Kriging-predicted residual and logistic regression-scored trend component. Percent change of RMSPE comparing models adjusted for rater to those not rater-adjusted were calculated; negative\% change of RMSPE indicating that adjustment for rater yielded lower audit item predictive error. Area Under the Curve (AUC) of the Receiver Operator Curve (ROC) of the validation datasets were calculated to assess the overall predictive ability of the above modeling. In these ROC AUC calculations of the validation dataset, observed audit item response was the dependent variable and predicted response probability (e.g., Kriging-predicted residual+ logistic regression-scored trend) was the single independent variable measured as a continuous variable. Percent change of ROC AUC comparing models adjusted for rater to those not rater-adjusted were calculated where, contrary to above, positive\% change of ROC 
Table 2 Small-scale spatial properties of neighborhood audit item responses, Essex County, NJ

\begin{tabular}{|c|c|c|c|c|c|c|c|c|}
\hline Audit item & Detrending & $\begin{array}{l}\text { Theoretical } \\
\text { semivariogram }\end{array}$ & Nugget & Partial sill/b & Range $(\mathrm{km}) / p$ & RMSPE & $\begin{array}{l}\text { \% RMSPE } \\
\text { change (of rater } \\
\text { adjustment) }\end{array}$ & $\begin{array}{l}\text { Evidence } \\
\text { of anisotropy }\end{array}$ \\
\hline \multirow[t]{2}{*}{ Garbage } & 3rd order spatial & Sine hole-Matern & 0.935 & $0.063,0.13$ & $4.731,0.871$ & 0.3940 & & None \\
\hline & $\begin{array}{c}\text { 3rd order spa- } \\
\text { tial + rater }\end{array}$ & Sine hole-Matern & 0.727 & $0.063,0.104$ & $4.385,0.795$ & 0.3376 & -14.31 & None \\
\hline \multirow[t]{2}{*}{ Abandoned Cars } & 3rd order spatial & $\begin{array}{l}\text { Sine hole-Sine } \\
\text { hole }\end{array}$ & 0 & $0.012,0.007$ & $1 E-6,1 E-6$ & 0.0491 & & NA \\
\hline & $\begin{array}{l}\text { 3rd order spa- } \\
\text { tial + rater }\end{array}$ & $\begin{array}{l}\text { Spherical-Sine } \\
\text { hole }\end{array}$ & $6.80 E-04$ & $0.16,0.002$ & $1 E-6,0.762$ & 0.0489 & -0.32 & NA \\
\hline \multirow{2}{*}{$\begin{array}{l}\text { Building condi- } \\
\quad \text { tions } \geq \text { moderate }\end{array}$} & 3rd order spatial & Power ${ }^{\mathrm{a}}$-Sine hole & 0.532 & $1 E-6,0.0247$ & $0.513,2.470$ & 0.2806 & & $\geq$ Mid-range \\
\hline & $\begin{array}{l}\text { 3rd order spa- } \\
\text { tial + rater }\end{array}$ & Sine hole-sine hole & $4.70 E-06$ & $0.025,0.493$ & $2.489,1 \mathrm{E}-6$ & 0.2704 & -3.63 & $\geq$ Mid-range \\
\hline \multirow{2}{*}{$\begin{array}{l}\text { Yard condi- } \\
\text { tions } \geq \text { moderate }\end{array}$} & 3rd order spatial & Sine hole-Matern & 0.459 & $0.045,0.019$ & $2.614,1 \mathrm{E}-6$ & 0.2596 & & $\geq$ Mid-range \\
\hline & $\begin{array}{l}\text { 3rd order spa- } \\
\text { tial + rater }\end{array}$ & Power ${ }^{\mathrm{a}}$-Sine hole & 0.429 & $1.4 \mathrm{E}-6,0.045$ & $0,2.655$ & 0.2455 & -5.44 & $\geq$ Mid-range \\
\hline \multirow[t]{2}{*}{ Dumpster } & 3rd order spatial & Matern-sine hole & 0.501 & $1.0 \mathrm{E}-6,0.007$ & $9.052,1.650$ & 0.2596 & & $\geq$ Mid-range \\
\hline & $\begin{array}{l}\text { 3rd order spa- } \\
\text { tial + rater }\end{array}$ & Sine hole-sine hole & 0.485 & $0.015,1.0 \mathrm{E}-6$ & $0.821,6.511$ & 0.2567 & -1.11 & $\geq$ Mid-range \\
\hline \multirow[t]{2}{*}{ Graffiti } & 3rd order spatial & Sine hole-Gaussian & 0.025 & $0.017,0.421$ & $1.020,1.0 \mathrm{E}-6$ & 0.2606 & & $\geq$ Mid-range \\
\hline & $\begin{array}{l}\text { 3rd order spa- } \\
\text { tial + rater }\end{array}$ & Matern-Sine hole & 0.433 & $6.3 \mathrm{E}-5,0.016$ & $10.141,1.028$ & 0.2566 & -1.54 & $\geq$ Mid-range \\
\hline \multirow{2}{*}{$\begin{array}{l}\text { Boarded/burned } \\
\text { building }\end{array}$} & 3rd order spatial & Cubic & 0.032 & 0.306 & 0.231 & 0.2138 & & $\geq$ Mid-range \\
\hline & $\begin{array}{l}\text { 3rd order spa- } \\
\text { tial + rater }\end{array}$ & Spherical-Matern & 0.004 & $1.0 \mathrm{E}-6,0.333$ & $4.085,0.094$ & 0.2140 & 0.12 & $\geq$ Mid-range \\
\hline \multirow[t]{2}{*}{ Outdoor seating } & 3rd order spatial & Sine hole & 1.131 & 0.015 & 4.405 & 0.4222 & & None \\
\hline & $\begin{array}{l}\text { 3rd order spa- } \\
\text { tial + rater }\end{array}$ & Sine hole-spherical & 0.006 & $0.011,1.089$ & $4.385,1.0 \mathrm{E}-6$ & 0.4078 & -3.41 & None \\
\hline \multirow[t]{2}{*}{ Team sports } & 3rd order spatial & Power ${ }^{\mathrm{a}}$-sine hole & 0.1658 & $8.6 \mathrm{E}-5,0.023$ & $2,6.4 \mathrm{E}-5$ & 0.1352 & & None \\
\hline & $\begin{array}{l}\text { 3rd order spa- } \\
\text { tial + rater }\end{array}$ & Power ${ }^{a}$ & 0.186 & $8.70 E-05$ & 2 & 0.1349 & -0.22 & None \\
\hline \multirow[t]{2}{*}{ Yard Decorations } & 3rd order spatial & Sine hole-gaussian & 1.095 & $0.046,0.146$ & $4.591,0.649$ & 0.4208 & & None \\
\hline & $\begin{array}{l}\text { 3rd order spa- } \\
\text { tial + rater }\end{array}$ & Sine hole-Matern & 0.023 & $0.048,1.141$ & $4.652,0.278$ & 0.4003 & -4.87 & None \\
\hline \multirow[t]{2}{*}{ Fences } & 3rd order spatial & $\begin{array}{l}\text { Gaussian-Expo- } \\
\text { nential }\end{array}$ & 0.65 & $0.109,0.200$ & $10.758,0.435$ & 0.3443 & & $\geq$ Mid-range \\
\hline & $\begin{array}{c}\text { 3rd order spa- } \\
\text { tial + rater }\end{array}$ & Sine hole-matern & $4.10 E-05$ & $0.064,0.826$ & $10.723,0.351$ & 0.3311 & -3.81 & $\geq$ Mid-range \\
\hline \multirow[t]{2}{*}{ Sidewalk present } & 3rd order spatial & Matern-sine hole & 0 & $0.904,0.069$ & $1.869,3.022$ & 0.2952 & & $\geq$ Mid-range \\
\hline & $\begin{array}{l}\text { 3rd order spa- } \\
\text { tial + rater }\end{array}$ & Power ${ }^{\mathrm{a}}$-matern & 0.558 & $2.70 \mathrm{E}-4,0.381$ & $2,1.683$ & 0.3035 & 2.8 & $\geq$ Mid-range \\
\hline \multirow[t]{2}{*}{ Complete sidewalk } & 3rd order spatial & Gaussian-sine hole & 0.443 & $0.164,0.015$ & $7.648,2.542$ & 0.2512 & & None \\
\hline & $\begin{array}{l}\text { 3rd order spa- } \\
\text { tial + rater }\end{array}$ & Gaussian-spherical & 0.432 & $0.107,0.070$ & $8.959,10.3981$ & 0.2511 & -0.01 & None \\
\hline \multirow[t]{2}{*}{ Sidewalk condition } & 3rd order spatial & Gaussian-Sine hole & 1.225 & $1.8 \mathrm{E}-6,0.029$ & $1.0 \mathrm{E}-6,2.409$ & 0.4403 & & None \\
\hline & $\begin{array}{l}\text { 3rd order spa- } \\
\text { tial + rater }\end{array}$ & $\begin{array}{l}\text { Exponential-sine } \\
\text { hole }\end{array}$ & 1.106 & $1.0 \mathrm{E}-6,0.034$ & $4.139,2.495$ & 0.4297 & -2.39 & None \\
\hline \multirow[t]{2}{*}{ Sidewalk width } & 3rd order spatial & $\begin{array}{l}\text { Gaussian-expo- } \\
\text { nential }\end{array}$ & 0.019 & $0.637,0.005$ & $0.099,1.0 \mathrm{E}-6$ & 0.2870 & & None \\
\hline & $\begin{array}{l}\text { 3rd order spa- } \\
\text { tial + rater }\end{array}$ & Cubic-matern & 0.11 & $1.0 \mathrm{E}-6,0.538$ & $4.782,0.109$ & 0.2779 & -3.18 & None \\
\hline \multirow[t]{2}{*}{$\begin{array}{l}\text { Sidewalk from } \\
\text { Curb Distance }\end{array}$} & 3rd order spatial & $\begin{array}{l}\text { Sine hole-Spher- } \\
\text { ical }\end{array}$ & 0.04 & $0.076,0.011$ & $1.0 \mathrm{E}-6,3.918$ & 0.0938 & & $\geq$ Mid-range \\
\hline & $\begin{array}{l}\text { 3rd order spa- } \\
\text { tial + rater }\end{array}$ & $\begin{array}{l}\text { Sine hole-expo- } \\
\text { nential }\end{array}$ & 0.036 & $0.012,0.076$ & $3.953,1.0 \mathrm{E}-6$ & 0.0936 & -0.15 & $\geq$ Mid-range \\
\hline
\end{tabular}


Table 2 (continued)

\begin{tabular}{|c|c|c|c|c|c|c|c|c|}
\hline Audit item & Detrending & $\begin{array}{l}\text { Theoretical } \\
\text { semivariogram }\end{array}$ & Nugget & Partial sill/b & Range $(\mathrm{km}) / p$ & RMSPE & $\begin{array}{l}\text { \% RMSPE } \\
\text { change (of rater } \\
\text { adjustment) }\end{array}$ & $\begin{array}{l}\text { Evidence } \\
\text { of anisotropy }\end{array}$ \\
\hline \multirow[t]{2}{*}{ Car Obstruction } & 3rd order spatial & Gaussian-cubic & 0.09 & $9.5 E-4,0.320$ & $1.0 \mathrm{E}-6,0.251$ & 0.2003 & & $\geq$ Mid-range \\
\hline & $\begin{array}{l}\text { 3rd order spa- } \\
\text { tial + rater }\end{array}$ & Spherical-spherical & 0.006 & $0.368,0.034$ & $0.208,1.0 \mathrm{E}-6$ & 0.1996 & -0.32 & $\geq$ Mid-range \\
\hline \multirow{2}{*}{$\begin{array}{l}\text { Garbage can } \\
\text { obstruction }\end{array}$} & 3rd order spatial & Power $^{\mathrm{a}}$ & 0.388 & $1.60 \mathrm{E}-04$ & 2 & 0.1979 & & None \\
\hline & $\begin{array}{l}\text { 3rd order spa- } \\
\text { tial + rater }\end{array}$ & Power ${ }^{\mathrm{a}}$-sine hole & 0.372 & $1.4 \mathrm{E}-4,0.014$ & $2,1.0 \mathrm{E}-6$ & 0.1974 & -0.27 & None \\
\hline \multirow{2}{*}{$\begin{array}{l}\text { Pole or Sign } \\
\text { obstruction }\end{array}$} & 3rd order spatial & Power ${ }^{a}$ & 0.338 & $5.90 E-04$ & 2 & 0.1620 & & $\geq$ Mid-range \\
\hline & $\begin{array}{l}\text { 3rd order spa- } \\
\text { tial + rater }\end{array}$ & Power ${ }^{\mathrm{a}}$-sine hole & 0.018 & $6.2 E-5,0.298$ & $2,1.0 \mathrm{E}-6$ & 0.1624 & 0.29 & $\geq$ Mid-range \\
\hline \multirow[t]{2}{*}{ Other obstruction } & 3rd order spatial & Spherical-gaussian & $6.10 E-04$ & $2.2 \mathrm{E}-4,0.495$ & $1.0 \mathrm{E}-6,0.067$ & 0.2450 & & None \\
\hline & $\begin{array}{l}\text { 3rd order spa- } \\
\text { tial + rater }\end{array}$ & Matern-matern & $2.80 E-04$ & $1.6 \mathrm{E}-4,0.491$ & $1.0 \mathrm{E}-6,0.068$ & 0.2427 & -0.93 & None \\
\hline \multirow[t]{2}{*}{ Curb Cuts } & 3rd order spatial & Matern-sine hole & $9.00 E-09$ & $1.345,0.026$ & $0.094,4.131$ & 0.4751 & & None \\
\hline & $\begin{array}{l}\text { 3rd order spa- } \\
\text { tial + rater }\end{array}$ & Sine hole-Power ${ }^{a}$ & 1.186 & $6.1 E-3,0.030$ & $4.303,0.360$ & 0.4587 & -3.45 & None \\
\hline \multirow[t]{2}{*}{ Clear Intersection } & 3rd order spatial & Sine hole-Matern & $3.30 E-05$ & $0.028,1.272$ & $12.078,0.409$ & 0.4148 & & None \\
\hline & $\begin{array}{l}\text { 3rd order spa- } \\
\text { tial + rater }\end{array}$ & Sine hole-matern & $9.20 E-05$ & $0.028,1.271$ & $11.815,0.410$ & 0.4143 & -0.12 & None \\
\hline \multirow{2}{*}{$\begin{array}{l}\text { Pedestrian Cross- } \\
\text { ing Sign }\end{array}$} & 3rd order spatial & Spherical-Cubic & 0.383 & $1.0 \mathrm{E}-6,0.494$ & $6.008,0.258$ & 0.3739 & & None \\
\hline & $\begin{array}{l}\text { 3rd order spa- } \\
\text { tial + rater }\end{array}$ & Spherical-Matern & 0.242 & $1.0 \mathrm{E}-3,0.630$ & $1.0 \mathrm{E}-6,8.550$ & 0.3747 & 0.2 & None \\
\hline \multirow[t]{2}{*}{ Pedestrian Signal } & 3rd order spatial & Spherical-matern & 0.063 & $6.8 \mathrm{E}-4,0.713$ & $1.0 \mathrm{E}-6,0.148$ & 0.3348 & & $\geq$ Mid-range \\
\hline & $\begin{array}{c}\text { 3rd order spa- } \\
\text { tial + rater }\end{array}$ & Matern-matern & 0.117 & $4.5 E-3,0.646$ & $8.045,0.1523$ & 0.3326 & -0.67 & $\geq$ Mid-range \\
\hline \multirow{2}{*}{$\begin{array}{l}\text { Pedestrian crossing } \\
\text { marks }\end{array}$} & 3rd order spatial & Sine hole-Matern & 0.649 & $0.074,0.416$ & $8.039,0.991$ & 0.3634 & & $\geq$ Mid-range \\
\hline & $\begin{array}{l}\text { 3rd order spa- } \\
\text { tial + rater }\end{array}$ & Sine hole-matern & 0.597 & $0.068,0.447$ & $7.660,0.988$ & 0.3676 & 1.15 & $\geq$ Mid-range \\
\hline \multirow{2}{*}{$\begin{array}{l}\text { Type of pedestrian } \\
\text { crosswalk marks }\end{array}$} & 3rd order spatial & Sine hole-gaussian & 0.669 & $0.053,0.457$ & $3.671,0.259$ & 0.3582 & & Yes, E-SE \\
\hline & $\begin{array}{l}\text { 3rd order spa- } \\
\text { tial + rater }\end{array}$ & Sine hole-matern & 0 & $0.052,1.124$ & $3.672,0.187$ & 0.3530 & -1.44 & Yes, E-SE \\
\hline \multirow[t]{2}{*}{ Traffic signal type } & 3rd order spatial & Sine hole-cubic & 0.8 & $0.119,0.215$ & $11.930,0.631$ & 0.3786 & & None \\
\hline & $\begin{array}{l}\text { 3rd order spa- } \\
\text { tial + rater }\end{array}$ & Matern-spherical & 0.779 & $0.174,0.226$ & $11.967,0.583$ & 0.3763 & -0.59 & None \\
\hline \multirow[t]{2}{*}{ One-way Street } & 3rd order spatial & $\begin{array}{l}\text { Exponential- } \\
\text { matern }\end{array}$ & 0.081 & $1.0 \mathrm{E}-6,0.462$ & $6.700,0.146$ & 0.2397 & & $\geq$ Mid-range \\
\hline & $\begin{array}{l}\text { 3rd order spa- } \\
\text { tial + rater }\end{array}$ & $\begin{array}{l}\text { Sine hole-expo- } \\
\text { nential }\end{array}$ & 0.005 & $0.041,0.491$ & $0.182,0.100$ & 0.2412 & 0.62 & $\geq$ Mid-range \\
\hline \multirow[t]{2}{*}{ Number of lanes } & 3rd order spatial & $\begin{array}{l}\text { Exponential-sine } \\
\text { hole }\end{array}$ & 0.493 & $0.188,0.057$ & $0.690,2.295$ & 0.3066 & & $\geq$ Mid-range \\
\hline & $\begin{array}{l}\text { 3rd order spa- } \\
\text { tial + rater }\end{array}$ & Matern-spherical & 0.22 & $0.364,0.155$ & $0.210,3.020$ & 0.2880 & -6.09 & $\geq$ Mid-range \\
\hline \multirow{2}{*}{$\begin{array}{l}\text { Presence of } \\
\text { highway }\end{array}$} & 3rd order spatial & Power ${ }^{\mathrm{a}}$-matern & 0.126 & $1.9 E-4,0.236$ & $2,0.738$ & 0.1611 & & $\geq$ Mid-range \\
\hline & $\begin{array}{l}\text { 3rd order spa- } \\
\text { tial + rater }\end{array}$ & Power ${ }^{\mathrm{a}}$-matern & 0.126 & $1.9 E-4,0.236$ & $2,0.737$ & 0.1616 & 0.31 & $\geq$ Mid-range \\
\hline \multirow[t]{2}{*}{ Highway is barrier } & 3rd order spatial & Cubic-sine hole & 0 & $0.772,0.206$ & $0.412,2.597$ & 0.4451 & & $\geq$ Mid-range \\
\hline & $\begin{array}{l}\text { 3rd order spa- } \\
\text { tial + rater }\end{array}$ & Sine hole-Gaussian & 0.043 & $0.180,0.641$ & $2.439,1.0 \mathrm{E}-6$ & 0.4690 & 5.37 & $\geq$ Mid-range \\
\hline
\end{tabular}

All kriging was local, with radius set at $1.3 \mathrm{~km}$ (approx 1/20th of largest distance between points) or the shortest distance to include 30 neighbors in Kriging calculations

a Power semivariogram $b$ and $p$ are not partial sill and range values and cannot be compared to values of other semivariograms 
AUC indicated that adjustment for rater yielded greater audit item predictive ability. The following interpretations of ROC AUC accuracy were used: $\mathrm{ROC}=0.5$ 'None', $0.7 \leq \mathrm{ROC}$ AUC $<0.8$ 'Acceptable', $0.8 \leq \mathrm{ROC}$ AUC $<0.9$ 'Excellent', ROC AUC $\geq 0.9$ 'Outstanding' [57].

Lastly, correlations between predicted audit item responses and select block group-level census variables were calculated to explore relationships between various audit items, sociodemographic, and neighborhood features. Audit item response predictions were based on the full model and a separate set of 10 locations randomly generated within each of Essex County's 671 block groups (i.e., 6710 prediction locations). As correlations were block group-level, the 10 audit item response values per a block group were treated as imputed data and analyzed within a multiple imputation framework, as has been previously done [27]. Block group-level percentage of non-Hispanic African American residents (\% AA), percentage of HispanicLatinx residents /(\% Latinx), percentage of non-Hispanic White residents (\% NHW), percentage of residents who moved within the previous year, percentage of working-age people who walk to work, median year of homes' construction, median gross rent, median owner occupied home value, and population density were from the 2011-2015 American Community Survey [58]. Data management and analyses were conducted within 64-bit, desktop versions of ArcGIS v10.5, SAS v9.4, and the Spatstat package within R v3.5.2 [59-61]. All spatial data were projected Alber's Equidistant Conic to preserve accurate distance calculations.

\section{Results}

Spatially varying probability surfaces of each audit item's response indicate largely unique geographic patterns across audit items. There are, however, notable trends especially when audit item response patterns are considered within item groupings. Presence of garbage, abandoned cars, $<$ moderate building or yard conditions, dumpsters, graffiti, and boarded up/burned out buildings appear to be concentrated in the southeast portion of the study region (Newark), and hence, appear to correlate with another (Fig. 3a-g). Presence of outdoor seating, team sports in public spaces, and yard decorations tend to occur at lower than average probabilities within the southeast, but also appear to be highly variable throughout the remainder of the study region. Sidewalk presence and complete sidewalks were estimated to be higher towards the eastern portion of the region. Among areas with sidewalks, sidewalks of good condition tended to be less commonly found within the southeast. Sidewalks wider than 4 feet were more likely in the eastern portions. Sidewalks obstructed by either a car, pole or sign, or something else (besides garbage can) were more likely in the southeast of the study region. Presence of an intersection was concentrated in the more densely populated eastern section as was presence of pedestrian crossing signs, signals, and marked crosswalks.

Results of theoretical semivariogram fitting by weighted least squares regression are shown in Table 2. There are several general results worth noting from these fitted semivariograms of the detrended audit items. First, the majority of audit items were best estimated, in terms of lower sums of squares error, by nested semivariograms as opposed to a single theoretical semivariogram. The better fitting nested semivariograms indicate that audit item responses spatially autocorrelate at more than one scale. For example, the best fitting semivariograms to the 3rd order spatially-detrended response pattern of the 'Garbage' audit item suggests a sharp rise in semivariance from an initial nugget of 0.935 to a semivariance of 1.065 (Matern partial sill of 0.13) over a distance of $0.871 \mathrm{~km}$ (Matern range), then a second and flatter rise over a distance of $4.731 \mathrm{~km}$ (sine hole range) until the sill $(1.128=0.935$ (nugget) +0.13 (Matern partial sill) +0.063 (sine hole partial sill)). Second, a majority (21/31) of theoretical semivariogram nuggets of audit items additionally detrended for 'rater' were lower than theoretical semivariogram nuggets of audit items detrended only for 3rd order spatial relationships. As the nugget is a measure of measurement error or short-distance spatial heterogeneity, lower nuggets of rater-detrended audit item responses could indicate that rater disagreement in item responses (e.g., lower test-retest and inter-rater reliability) might increase short-distance measurement error. Third, RMSPE of rater-adjusted audit item responses were lower than RMSPE of responses adjusted for spatial trend only in 23 of 31 audit items, indicating that rater adjustment of these 23 audit items resulted in improved prediction accuracy via the cross-validation Kriging models. Fourth, several audit item experimental semivariogams best fit by nested theoretical semivariograms indicate a small nugget which coincides with a large 1st partial sill over a very small range followed by a 2nd, smaller partial sill and larger range. Audit item semivariograms characterized this way have at least one short-distance binned empirical semivariogram value with markedly low semivariance (i.e., high correlation at short distance), which leads to a fitted theoretical semivariogram that is "bent" downwards by the influence of the low variance values (Additional file 1: Figs. S1a-ae 1) and 2)). Fifth, there was no evidence of anisotropy among 13 audit items-4 neighborhood physical disorderrelated, 6 sidewalk-related, 3 intersection-related-and evidence of anisotropy " $\geq$ Mid-range" among 16 items (Additional file 1: Figs. S2a-ae 1) and 2)). As an example of potential $\geq$ Mid-range anisotropy, the sills $(\sim 0.6)$ 


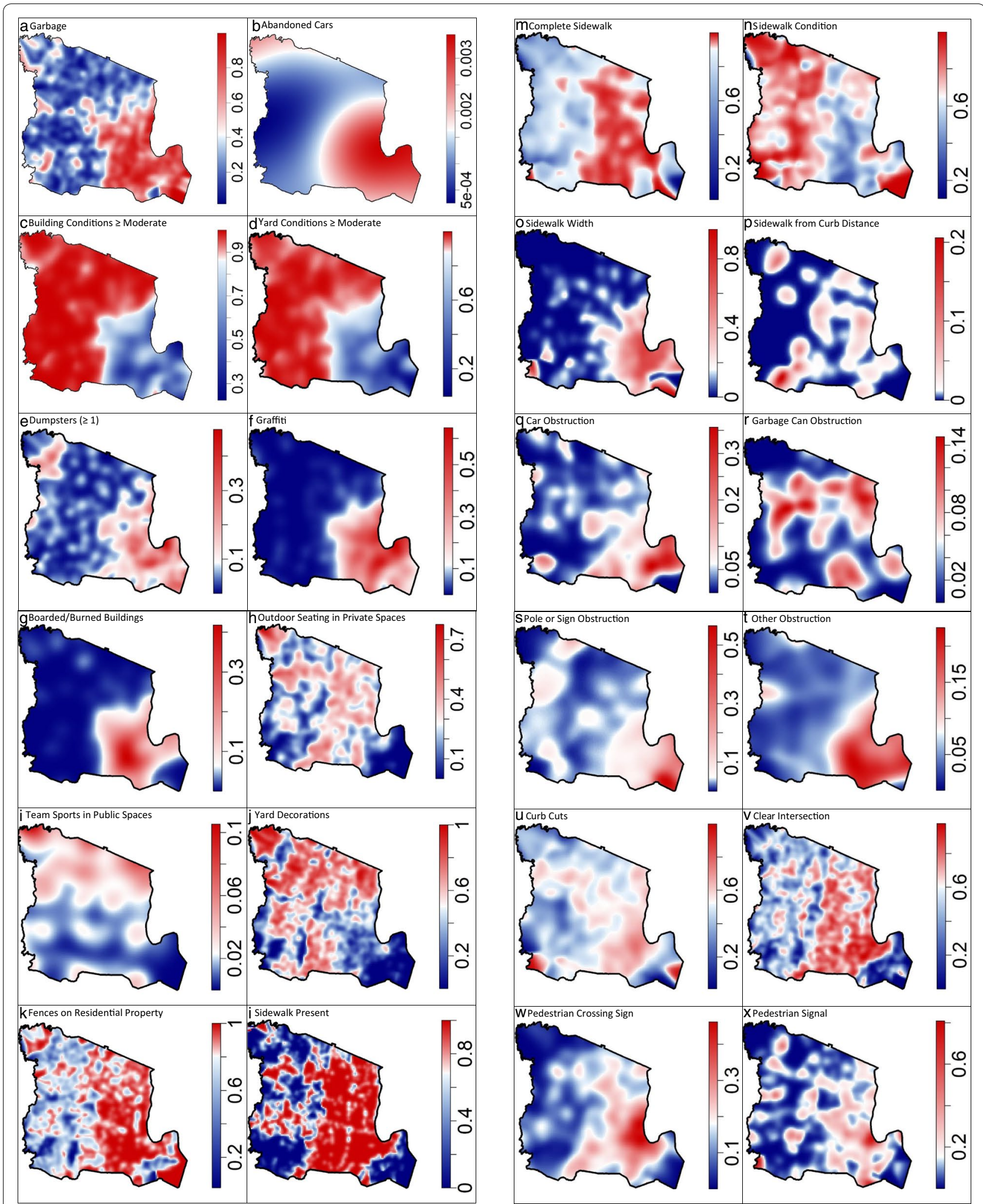

Fig. 3 a-ae Nonparametric, spatially varying probability surfaces of each audit item response ="Yes"/"1", Essex County, NJ'. ${ }^{1}$ Shaded via a divergent color scheme with red and blue hue proportional to estimated probability of $Y e s^{\prime} /{ }^{\prime} 1$ ' and white equal to the overall probability of each item (Table 1) 


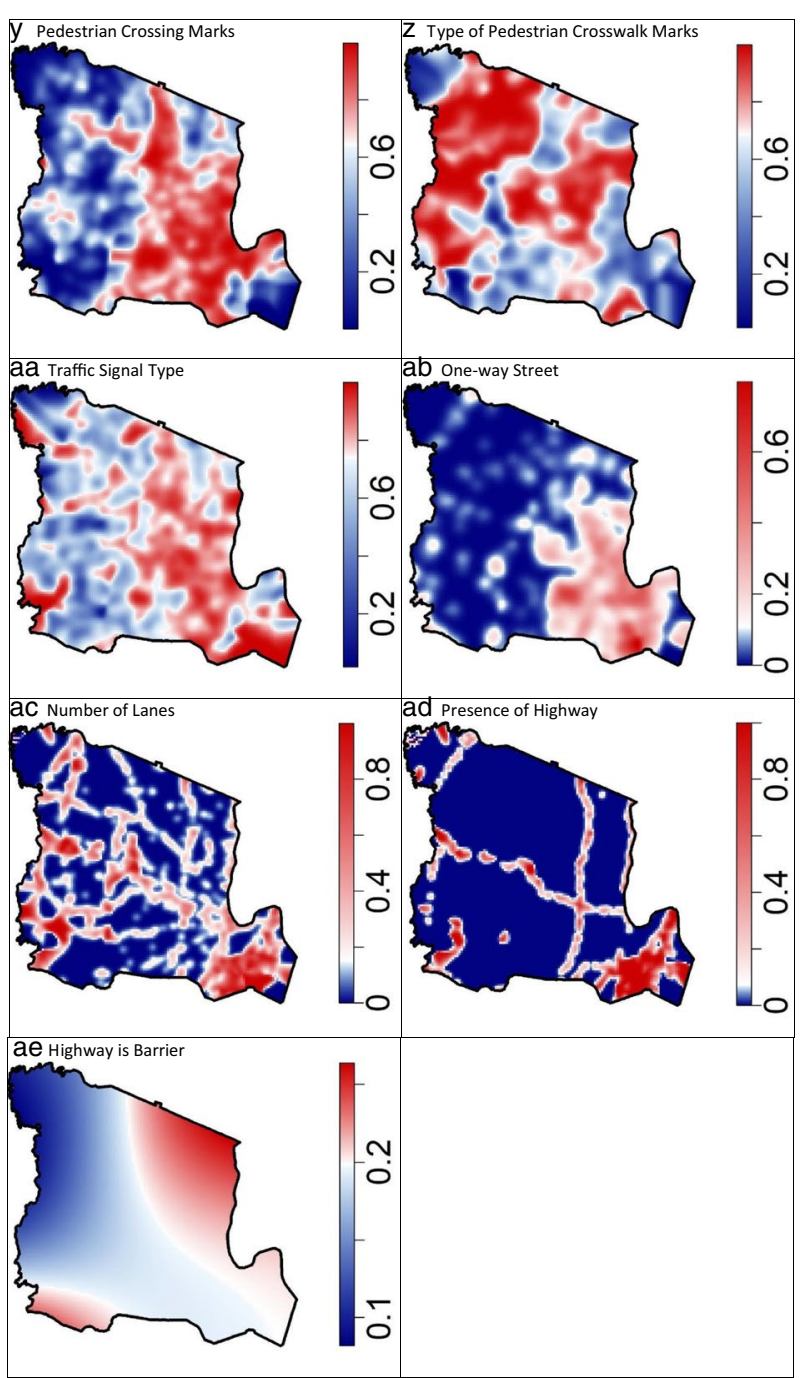

Fig. 3 continued

and semivariogram ranges $(\sim 2.4-3.5 \mathrm{~km})$ look nearly identical across all eight directional semivariograms of the "Building conditions $\geq$ Moderate" item (Additional file 1: Fig. S2c.1)) and are similar to the estimated sill $(0.56)$ and range $(1 \mathrm{st}=0.513 \mathrm{~km}, 2 \mathrm{nd}=2.470 \mathrm{~km})$ of the omnidirectional semivariogram reported in Table 2. However, certain directional semivariances decrease as distance increases beyond these ranges (directional semivariograms of angles $0^{\circ} / 180^{\circ}, 22.5^{\circ} / 202.5^{\circ}, 45^{\circ} / 225^{\circ}$, $67.5^{\circ} / 247.5^{\circ}$ ), while others exhibit cyclical variability beyond these ranges $\left(90^{\circ} / 270^{\circ}, 112.5^{\circ} / 292.5^{\circ}, 135^{\circ} / 315^{\circ}\right)$, and one is flat beyond the range $\left(157.5^{\circ} / 337.5^{\circ}\right)$.

Table 3 displays the predictive accuracy of large spatial scale, small spatial scale, and rater components, as symbolized in the above equation. Regardless of rater adjustment, additional modeling of small-scale spatial relationships via Kriging results in a markedly higher
ROC AUC of observed audit item responses for all audit items compared to modeling only large-scale relationships. When considering all 62 full models (e.g., large-scale + small-scale spatial modeling with or without rater adjustment), 29 models -12 neighborhood physical disorder, 9 intersection-related, 8 sidewalkrelated-had 'outstanding' predictive accuracies (ROC AUC $\geq 0.9$ ), indicating that $90 \%$ of observed responses for these audit items of the validation dataset could be classified as 'Yes'/'1' or 'No'/'2' based on predictions from the training dataset. Only 1 model-highway is a barrier including rater adjustment-resulted in a less than 'Acceptable' predictive accuracy (ROC $\mathrm{AUC}=0.583$ ).

A majority of audit items' (18/31) predictive accuracy improved with additional adjustment for rater within the system of equations. There was variation in predictive 
Table 3 Prediction ability of neighborhood audit item responses by spatial scale (large/small) and rater adjustment

\begin{tabular}{|c|c|c|c|c|c|}
\hline \multirow[t]{2}{*}{ Audit item } & \multirow[t]{2}{*}{ Detrending } & \multicolumn{4}{|c|}{ ROC AUC prediction* } \\
\hline & & $\begin{array}{l}\text { Large-scale/ } \\
\text { Rater }\end{array}$ & $\begin{array}{l}\text { + Small-scale } \\
\text { Kriging }\end{array}$ & $\begin{array}{l}\text { \%Change of rater } \\
\text { adjustment, full equation }\end{array}$ & $\begin{array}{l}\text { \%Change large- } \\
\text { vs small-scale }\end{array}$ \\
\hline \multirow[t]{2}{*}{ Garbage } & 3rd order spatial & 0.755 & 0.856 & & 13.4 \\
\hline & 3rd order spatial + rater & 0.874 & 0.919 & 7.36 & 5.1 \\
\hline \multirow[t]{2}{*}{ Abandoned cars } & 3rd order spatial & 0.881 & 0.996 & & 13.1 \\
\hline & 3rd order spatial + rater & 0.952 & 0.994 & -0.2 & 4.4 \\
\hline \multirow[t]{2}{*}{ Building conditions $\geq$ moderate } & 3rd order spatial & 0.852 & 0.902 & & 5.9 \\
\hline & 3rd order spatial + rater & 0.880 & 0.916 & 1.55 & 4.1 \\
\hline \multirow[t]{2}{*}{ Yard conditions $\geq$ moderate } & 3rd order spatial & 0.897 & 0.934 & & 4.1 \\
\hline & 3rd order spatial + rater & 0.921 & 0.946 & 1.28 & 2.7 \\
\hline \multirow[t]{2}{*}{ Dumpster } & 3rd order spatial & 0.714 & 0.842 & & 17.9 \\
\hline & 3rd order spatial + rater & 0.714 & 0.849 & 0.83 & 18.9 \\
\hline \multirow[t]{2}{*}{ Graffiti } & 3rd order spatial & 0.871 & 0.914 & & 4.9 \\
\hline & 3rd order spatial + rater & 0.887 & 0.923 & 0.98 & 4.1 \\
\hline \multirow[t]{2}{*}{ Boarded/burned building } & 3rd order spatial & 0.851 & 0.914 & & 7.4 \\
\hline & 3rd order spatial + rater & 0.850 & 0.915 & 0.11 & 7.6 \\
\hline \multirow[t]{2}{*}{ Outdoor seating } & 3rd order spatial & 0.562 & 0.735 & & 30.8 \\
\hline & 3rd order spatial + rater & 0.679 & 0.767 & 4.35 & 13 \\
\hline \multirow[t]{2}{*}{ Team sports } & 3rd order spatial & 0.591 & 0.915 & & 54.8 \\
\hline & 3rd order spatial + rater & 0.683 & 0.894 & -2.3 & 30.9 \\
\hline \multirow[t]{2}{*}{ Yard decorations } & 3rd order spatial & 0.667 & 0.825 & & 23.7 \\
\hline & 3rd order spatial + rater & 0.708 & 0.852 & 3.27 & 20.3 \\
\hline \multirow[t]{2}{*}{ Fences } & 3rd order spatial & 0.710 & 0.884 & & 24.5 \\
\hline & 3rd order spatial + rater & 0.736 & 0.897 & 1.47 & 21.9 \\
\hline \multirow[t]{2}{*}{ Sidewalk present } & 3rd order spatial & 0.814 & 0.950 & & 16.7 \\
\hline & 3rd order spatial + rater & 0.814 & 0.943 & -0.74 & 15.8 \\
\hline \multirow[t]{2}{*}{ Complete sidewalk } & 3rd order spatial & 0.683 & 0.842 & & 23.3 \\
\hline & 3rd order spatial + rater & 0.726 & 0.855 & 1.54 & 17.8 \\
\hline \multirow[t]{2}{*}{ Sidewalk condition } & 3rd order spatial & 0.662 & 0.771 & & 16.5 \\
\hline & 3rd order spatial + rater & 0.697 & 0.775 & 0.52 & 11.2 \\
\hline \multirow[t]{2}{*}{ Sidewalk width } & 3rd order spatial & 0.830 & 0.897 & & 8.1 \\
\hline & 3rd order spatial + rater & 0.831 & 0.901 & 0.45 & 8.4 \\
\hline \multirow[t]{2}{*}{ Sidewalk from curb distance } & 3rd order spatial & 0.586 & 0.978 & & 66.9 \\
\hline & 3rd order spatial + rater & 0.724 & 0.914 & -6.54 & 26.2 \\
\hline \multirow[t]{2}{*}{ Car obstruction } & 3rd order spatial & 0.626 & 0.879 & & 40.4 \\
\hline & 3rd order spatial + rater & 0.663 & 0.881 & 0.23 & 32.9 \\
\hline \multirow[t]{2}{*}{ Garbage can obstruction } & 3rd order spatial & 0.674 & 0.929 & & 37.8 \\
\hline & 3rd order spatial + rater & 0.695 & 0.917 & -1.29 & 31.9 \\
\hline \multirow[t]{2}{*}{ Pole or sign obstruction } & 3rd order spatial & 0.592 & 0.900 & & 52 \\
\hline & 3rd order spatial + rater & 0.726 & 0.876 & -2.67 & 20.7 \\
\hline Other obstruction & 3rd order spatial & 0.689 & 0.870 & & 26.3 \\
\hline & 3rd order spatial + rater & 0.694 & 0.871 & 0.11 & 25.5 \\
\hline Curb cuts & 3rd order spatial & 0.559 & 0.710 & & 27 \\
\hline & 3rd order spatial + rater & 0.669 & 0.716 & 0.85 & 7 \\
\hline Clear intersection & 3rd order spatial & 0.681 & 0.851 & & 25 \\
\hline & 3rd order spatial + rater & 0.684 & 0.852 & 0.12 & 24.6 \\
\hline Pedestrian crossing sign & 3rd order spatial & 0.669 & 0.827 & & 23.6 \\
\hline & 3rd order spatial + rater & 0.664 & 0.809 & -2.18 & 21.8 \\
\hline
\end{tabular}


Table 3 (continued)

\begin{tabular}{|c|c|c|c|c|c|}
\hline \multirow[t]{2}{*}{ Audit item } & \multirow[t]{2}{*}{ Detrending } & \multicolumn{4}{|c|}{ ROC AUC prediction* } \\
\hline & & $\begin{array}{l}\text { Large-scale/ } \\
\text { Rater }\end{array}$ & $\begin{array}{l}\text { + Small-scale } \\
\text { Kriging }\end{array}$ & $\begin{array}{l}\text { \%Change of rater } \\
\text { adjustment, full equation }\end{array}$ & $\begin{array}{l}\text { \%Change large- } \\
\text { vs small-scale }\end{array}$ \\
\hline \multirow[t]{2}{*}{ Pedestrian signal } & 3rd order spatial & 0.651 & 0.908 & & 39.5 \\
\hline & 3rd order spatial + rater & 0.679 & 0.897 & -1.21 & 32.1 \\
\hline \multirow[t]{2}{*}{ Pedestrian crossing marks } & 3rd order spatial & 0.731 & 0.871 & & 19.2 \\
\hline & 3rd order spatial + rater & 0.725 & 0.860 & -1.26 & 18.6 \\
\hline \multirow[t]{2}{*}{ Type of pedestrian crosswalk marks } & 3rd order spatial & 0.697 & 0.931 & & 33.6 \\
\hline & 3rd order spatial + rater & 0.709 & 0.934 & 0.32 & 31.7 \\
\hline \multirow[t]{2}{*}{ traffic signal type } & 3rd order spatial & 0.661 & 0.841 & & 27.2 \\
\hline & 3rd order spatial + rater & 0.699 & 0.838 & -0.36 & 19.9 \\
\hline \multirow[t]{2}{*}{ One-way street } & 3rd order spatial & 0.789 & 0.931 & & 18 \\
\hline & 3rd order spatial + rater & 0.793 & 0.923 & -0.86 & 16.4 \\
\hline \multirow[t]{2}{*}{ Number of lanes } & 3rd order spatial & 0.676 & 0.920 & & 36.1 \\
\hline & 3rd order spatial + rater & 0.674 & 0.948 & 3.04 & 40.7 \\
\hline \multirow[t]{2}{*}{ Presence of highway } & 3rd order spatial & 0.838 & 0.993 & & 18.5 \\
\hline & 3rd order spatial + rater & 0.838 & 0.993 & 0 & 18.5 \\
\hline \multirow[t]{2}{*}{ Highway is barrier } & 3rd order spatial & 0.563 & 0.833 & & 48 \\
\hline & 3rd order spatial + rater & 0.479 & 0.583 & -30.01 & 21.7 \\
\hline
\end{tabular}

*The area under the curve (AUC) of receiver operator curves $(\mathrm{ROC})$ resulting from each item-specific logistic regression of the validation dataset was calculated where observed audit item response was the dependent variable and predicted response probability was the single independent variable measured as a continuous variable

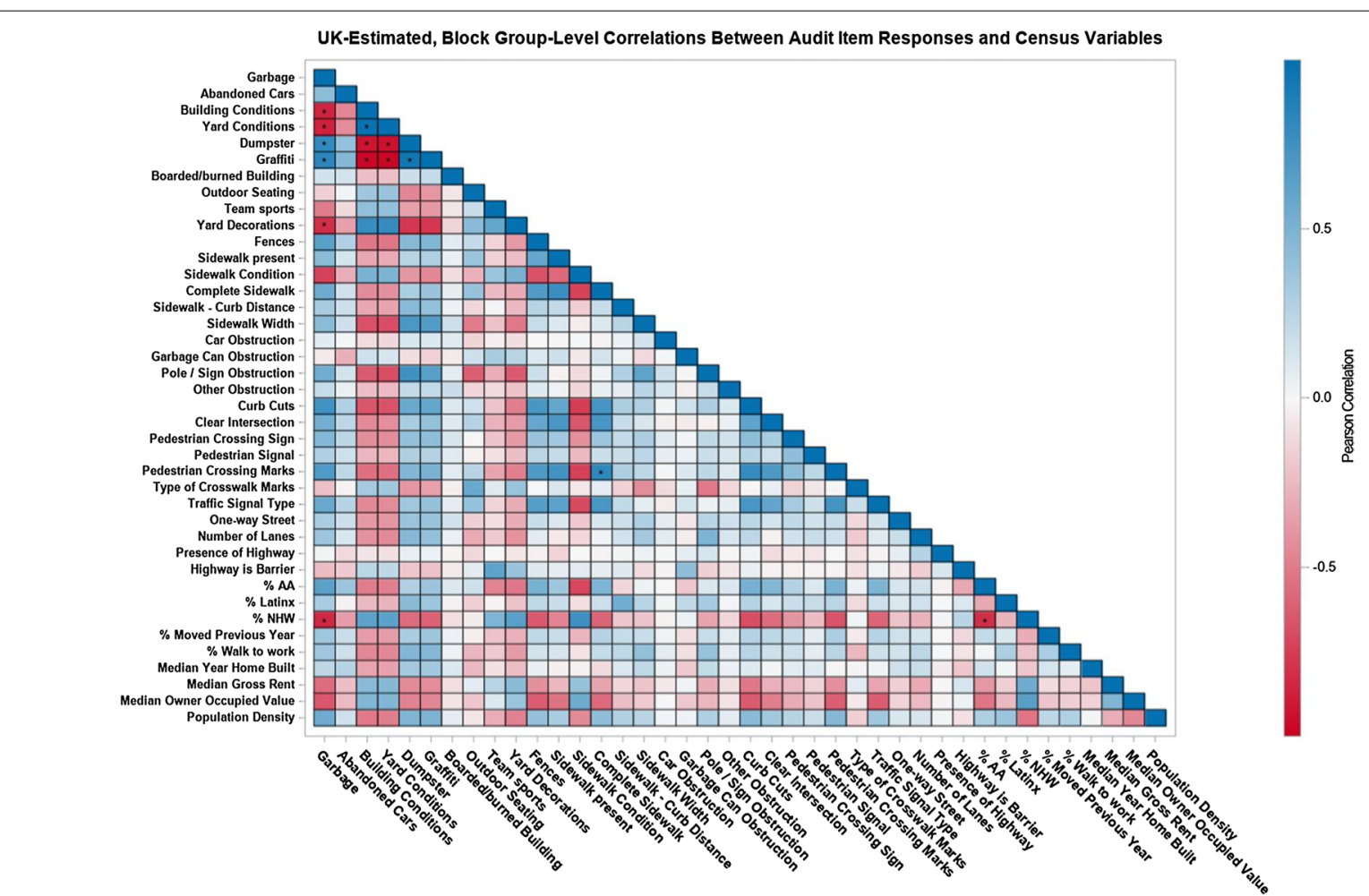

Fig. 4 Correlation matrix of block group-level, predicted audit item responses and sociodemographic, economic, and housing characteristics ${ }^{1,2}$. ${ }^{1}$ From the 2011-2015 American Community Survey. ${ }^{2}$ Pearson correlations greater than $|0.8|$ noted with an asterisk 
accuracy improvement by audit item grouping with 9 of 11 neighborhood physical disorder audit items, 6 of 10 sidewalk-related, and 3 of 10 intersection-related audit items indicating improved prediction accuracy with rater adjustment. Percent improvement in ROC AUC with adjustment for rater ranges from a low of $-30.01 \%$ (Highway is Barrier, worsening prediction) to $7.36 \%$ (Garbage, improved prediction). When considering the worse predictive ability of "Highway is Barrier" as an outlier (next worse is $-6.54 \%$ ), the overall average $\%$ improvement in ROC AUC with rater adjustment is $0.34 \%$ - neighborhood physical disorder-related item average $=1.7 \%$, sidewalk-related item average $=-0.3 \%$, intersection-related item average $=-0.6 \%$. All 62 models that accounted for small-scale spatial variation resulted in improved spatial prediction accuracy. The largest improvements associated with small-scale spatial modeling were among sidewalk- and intersection-related items, indicating greater small-scale spatial variation in response patterns of these audit items.

Block group-level, pair-wise correlations between predicted audit item responses and sociodemographic, economic, and housing characteristics vary substantially in magnitude from near perfect correlation $(r=0.99$ building conditions-yard conditions) to near zero $(r=0.03$ outdoor seating-abandoned cars) (Fig. 4, Additional file 1: Table S1). Nine of the ten largest correlations (all $r \geq|0.83|$ ) involve combinations of five neighborhood physical disorder audit items-garbage, building conditions $\geq$ moderate, yard conditions $\geq$ moderate, graffiti, dumpsters-indicating their interdependence. The ten largest correlations involving census data $(|0.64| \leq r \leq|0.84|)$ include one of the three racial-ethnic composition variables with the five highest between presence of garbage and percentage non-Hispanic White $(r=-0.83)$, percentage non-Hispanic AA and percentage non-Hispanic White $(\mathrm{r}=-0.81)$, sidewalk conditions $\geq$ good and percentage non-Hispanic White $(r=0.77)$, sidewalk conditions $\geq$ good and percentage non-Hispanic AA $(r=-0.71)$, and curb cuts and percentage non-Hispanic White $(r=-0.68)$. The three largest correlations involving median owner occupied home value involved the same variables-percentage non-Hispanic White $(r=0.65)$, garbage $(r=-0.64)$, and sidewalk curb cuts $(r=-0.62)$-as the largest correlations involving median gross rent (percentage non-Hispanic White $r=0.56$, garbage $r=-0.56$, and sidewalk curb cuts $r=-0.51$ ). The five highest correlations involving percentage of residents who moved within the previous year $(|0.32| \leq r \leq|0.37|)$ were with garbage, building conditions $\geq$ were with building conditions $\geq$ moderate, yard conditions $\geq$ moderate, graffiti, dumpsters, and abandoned cars. The strongest correlates of the percentage of working-age people who walk to work also included dumpsters $(r=0.48)$, graffiti $(r=0.47)$, building conditions $\geq$ moderate $(r=-0.45)$, yard conditions $\geq$ moderate $(r=-0.44)$ along with sidewalk width $\geq 4$ feet $(r=0.41)$.

\section{Discussion}

The spatial properties (i.e., spatial trend, autocorrelation, predictive accuracy) of 32 built environment characteristics assessed via point-based virtual neighborhood audits vary by audit item, but nearly all spatial models predict audit responses with 'outstanding' accuracy. Sidewalk and intersection audit item responses tend to exhibit small-scale variability which indicate the need for samples that are more spatially dense compared to neighborhood physical disorder audit items. Correlations between predicted audit item response patterns and neighborhood factors indicate that block-group level neighborhood physical disorder-related items are most inter-dependent with one another as well as select sociodemographic, economic, and housing characteristics.

\section{Comparison with previous literature}

To the best of our knowledge, our work is the first to report extensively on spatial autocorrelation and spatial prediction on a diverse set of audit items over a large spatial scale. With a few notable exceptions [25, 27-30, 62-64], neighborhood audit studies, virtual or in-person, have not reported spatial properties of audited features [25, 27-30, 62-64]. Those studies reporting any spatial properties have mainly focused on investigations of spatial autocorrelation [25, 27-30, 62, 63], with only one known study investigating spatial prediction of four neighborhood disorder audit items (reporting no predictive performance metrics) [64], and no known studies reporting spatial trend properties. Studies reporting spatial autocorrelation have tested a mix of individual audit items [28-30, 62], and neighborhood physical disorder scores from data reduction techniques that yield single values per audit location from combinations of audit item responses [25, 27]. Spatial autocorrelation ranges of neighborhood physical disorder scale scores from previous studies of four major U.S. metropolitan areas varied from $1 \mathrm{~km}$ to $10 \mathrm{~km}[25,27]$. These ranges from neighborhood physical disorder scores were typically larger than ranges of individual audit items of neighborhood physical disorder-presence of buildings in disrepair $(0.72 \mathrm{~km})$ [28], presence of parcel-level gardens (range $\approx 0.61 \mathrm{~km}$ ) [29], block group-level garden density (range $\approx 0.40 \mathrm{~km}$ ) [30] - observed in other studies. Two additional studies tested the spatial autocorrelation of neighborhood physical disorder scores [63], and sidewalk completeness and width [62], but did not report the distances at which values spatially correlated. 


\section{Viability of point-based auditing}

Although comparisons to the few previous studies investigating spatial properties of audit responses are difficult, this study confirms and extends previous results in various ways. All 31 audit item response patterns demonstrated the presence of spatial autocorrelation, confirming previous studies of similar audit items or constructs. This study extends previous results in finding appreciable spatial autocorrelation based on 3rd order spatially-detrended audit item response patterns; estimated Kriging parameters (i.e., nugget, partial sill, range) are from audit item response patterns that are independent of larger-scale trends across the study area. The nonzero Kriging range parameters for all audit items parallels the improved spatial prediction accuracy of models that additionally adjust for small-scale spatial variation; smallscale spatial autocorrelation exists in these audit items. Also, of note was the pattern of best-fit, nested theoretical semivariograms (opposed to single semivariograms), suggesting that small-scale spatial variation operated at least two scales. Together, these results indicate that the spatial variability of audit item response patterns spatially autocorrelate across multiple scales, suggestive of multiple processes influencing these patterns. Factors that explain multi-scale spatial variation of audit item responses are likely specific to individual audit items or audit item construct grouping (e.g., all sidewalk-related items caused by common social processes). For example, the spatial correlation of yard conditions across a region might operate at multiple scales due to variation in individual and institutional economic resources or strongly related factors (e.g., disposable income for yard care equipment, number of foreclosed/real estate owned/ abandoned homes, municipal resources to care for public land, public/private disinvestment) and landscaping services (i.e., existence of such, affordability, universal servicer vs. multiple servicers coming on different days within the same street) might operate at a larger scale, whereas typical yard care practices of individuals and institutions occupying those regions or social network diffusion effects where presence of a well-kept yard influences neighbors to improve their yard (i.e., "landscape mimicry" $[29,30])$ might operate more locally.

As indicated in the results, the semivariograms best fit through nesting appear to be related to instances of a few binned empirical semivariogram values with markedly lower variance (i.e., higher correlation) at short distances. One the one hand it can be argued that the high correlation of audit item responses at short distances might be expected and reflective of social processes such as those detailed above for yard conditions. On the other, more statistically problematic hand, these highly correlated observations at short distances could be indicative of raters' characterizations of nearly-identical GSV scenes; akin to a Kriging analysis with duplicated observations which invalidates the results [65]. However, no two exact audit locations were rated more than once and less than $0.02 \%$ of semivariogram data points comprise the first two binned values of each audit item experimental semivariogram translating to very small contributions to the weighted least squares regression and fitted theoretical semivariogram. Pragmatically, it would very difficult to generate a sample of audit location points proximate enough to adequately test small-scale spatial variation while also ensuring that raters are not rating portions of the same scene more than once.

\section{Spatial model predictive accuracy}

That approximately half of full spatial models, regardless of rater adjustment, had at least $90 \%$ predictive accuracy - and $90 \%$ of models had at least $80 \%$ accuracy -suggests that this sample of point-based audit item responses were predicted well. Echoing the above discussion on audit item response multiscale spatial variation, additional modeling of small-scale spatial variation resulted in marked improvement in prediction accuracy for all items compared to large-scale spatial prediction alone, indicating the utility of regression Kriging for spatial prediction $[44,65]$. A main objective of this study was the exploration of spatial prediction performance variation due to systematic differences in rater test-retest and inter-rater agreement [32]. While a slim majority (18 of 31) of full spatial model's predictive accuracy improved with rater adjustment, patterns of prediction improvement might exist which could aid in guiding future analyses and decisions of whether to adjust for rater. For example, an overwhelming majority of neighborhood physical disorder-related, but minority of intersectionrelated, audit item responses saw improved prediction accuracy with statistical adjustment for rater. Comparing these spatial predictive accuracy patterns to previous reported patterns of rater agreement reliability seems to suggest that lower reliability items (neighborhood physical disorder-related) experience greater improvement in prediction accuracy compared to higher reliability items (intersection-related) when variation in rater is accounted for in statistical models [32]. Measurement theory conventionally partitions observed variation into a component attributable to true variation, a component attributable to systematic observer error and a component attributable to random error. It follows that, for lower reliability items (i.e. items in which more observed variation is attributable to error), there is more room for spatial improvement by adjusting for rater effects. Future validity studies of these audit items should test whether item or construct validity varies with rather adjustment 
in greater detail. Such validation studies will be helpful in deciding whether adjusting for rater is beneficial.

\section{Predicted audit item response correlations}

This study's findings of moderate-strong correlations among neighborhood physical disorder audit items and weak-moderate correlations between neighborhood physical disorder items and demographic, economic, and housing characteristics corroborate previous research $[3,25,27,28,63]$. Neighborhood physical disorder scores have been consistently built from visually audited assessments of items similar to those measured in this study: garbage/litter, empty liquor bottles, cigarettes in the street, graffiti, defaced property, abandoned cars, building conditions, deteriorated recreational spaces, boarded/burned buildings, vacant land, barred windows $[3,25,27,63]$. These studies have also found greater physical disorder to negatively correlate with area-level home or property value $[25,63]$, and positively correlate with individual-level AA race [28] and population density [27].

Although only correlational and not indicative of causal processes, relationships involving the three racial-ethnic density variables offer suggestions of areas for additional inquiry. New Jersey, and Essex County in particular, contains regions with some of the highest racial-ethnic residential segregation in the U.S. $[66,67]$. High correlations suggested that block groups with higher percentages of NHW residents have less garbage, more sidewalks in good condition, and fewer curb cuts. The latter correlation might be more indicative of NHW residents' tendency to live away from urban areas characterized by more intersections between roads and sidewalks, and hence curb cuts.

Other results of the correlation analysis deserving further attention, especially in future research involving physical disorder, is the moderate relationships between percentage of residents moving within the previous year and presence of garbage $(r=0.37)$, presence of graffiti $(\mathrm{r}=0.37)$, building conditions $\geq$ moderate $(\mathrm{r}=-0.36)$, yard conditions $\geq$ moderate $(r=-0.36)$, and presence of dumpsters $(r=0.32)$. These relationships suggest that block groups serving as destinations to greater proportions of recent residential movers are more likely to have higher physical disorder compared to areas with fewer residential movers. If holding under more rigorous analyses such results could inform research on neighborhood instability $[68,69]$, as well as underscore the importance of incorporating residential histories into studies involving built environment factors such as these [70-73].

Limitations of this study include the ad hoc regression Kriging method and uncertainty surrounding GSV as a reliable data source. Regression Kriging has been shown to generate estimates of the mean structure of a spatial process-large scale + small scale estimates - that are as accurate as Universal Kriging [44, 55]. However, most instances of regression Kriging involve linear, as opposed to logistic, regression of large-scale and covariate factors. No statistical methods exist within frequentist settings for Universal Kriging of binary data. Limitations of GSV data for assessment of built environment factorsunknown protocols for GSV driver routes, image acquisition, image processing, image updates; spatio-temporal patterns of image availability; suitability of environmental assessment of small or temporally variable items (e.g., garbage variation by day/time of day)-have been extensively detailed elsewhere [21, 22, 32, 74]. Of special relevance to this spatial analysis is the temporal variability of GSV scenes. An assumption of the spatial autocorrelation analyses is that temporal and spatial variability are independent of one another, which at least one previous study of spatio-temporal patterns of GSV image availability has brought into question [22]. For example, there is evidence that the GSV cars collect images in spatio-temporal batches based on whichever region the cars traverse [75], leading to spatially autocorrelated GSV image dates [22]. The influence of this relationship might could be mitigated if the spatio-temporal dependency is smooth across the study area. For example, GSV image batches that are collected and uploaded based on municipality adjacency, which would make economic sense from a transportation optimization perspective, would result in smooth changes in spatio-temporal patterns of GSV images. Regardless, these potential dependencies point to investigation of spatio-temporal prediction models of neighborhood audit responses [76], which coincide with the need mentioned above to investigate residential histories of individuals to whom GSV data will be linked in future studies. Finally, the audit items considered in this study were chosen based on their ability to be reliably observed and recorded through a standardized protocol, differentiating them from studies that prompt raters to provide their overall perception of a virtual scene's beauty, safety, or liveliness [74, 77]. Choosing between assessing a virtual scene for identifiable visual components versus a scene's perceived overall characteristics should not be based on whether one approach is generally superior to another, but rather the ultimate study question and planned translation of study findings. Identifying individually observable components of a virtual streetscape could motivate further studies and place-based interventions aimed at modifying the built environment as ways to improve population health [5]. 


\section{Conclusion}

Specific built environment- and physical disorder-related patterns assessed using a new point-based virtual neighborhood audit method spatially autocorrelate across multiple spatial scales, both short and longer distances, indicating the potential benefit of point-based over traditional, segment-based assessment methods. An overwhelming majority of audit item spatial patterns were well-predicted by regression Kriging spatial models, albeit with mixed results for whether statistical adjustment for rater response variability improves audit item spatial prediction. Predicted audit item responses related to physical disorder-garbage, graffiti, building conditions, yard conditions, boarded/abandoned buildings, and dumpsters,--were strongly related to one another as well as distributions of racial-ethnic composition, socioeconomic indicators, and residential mobility. Among these specific items, drop-and-spin virtual neighborhood auditing is a viable alternative to segment-based methodologies.

\section{Supplementary information}

Supplementary information accompanies this paper at https://doi. org/10.1186/s12942-020-00213-5.

Additional file 1. Supplementary methods of spatial analyses.

\section{Abbreviations}

GSV: Google Street View; U.S.: United States; NJ: New Jersey; GIS: Geographic Information System; CANVAS: Computer Assisted Neighborhood Virtual Assessment System; ROC: Receiver operator curves; AUC: Area under the curve; NA: Not applicable; OK: Ordinary Kriging; RMSPE: Root mean squared prediction error; AA: African American; NHW: Non-Hispanic White; km: Kilometer.

\section{Acknowledgements}

Not applicable.

\section{Authors' contributions}

Author JJP and SJM designed the study and directed its implementation, including quality assurance and control. Authors JJP, MS, AGR, CX, and SJM supervised data collection activities and designed the study's analytic strategy. Authors JJP, MS, AGR, CX, AAML, AMS and SJM drafted the work or substantively revised it, approved of the manuscript, agree both to be personally accountable for their own contributions and ensure that questions related to the accuracy or integrity of any part of the work, even ones in which they were not personally involved, are appropriately investigated, resolved, and the resolution documented in the literature. All authors read and approved the final manuscript.

\section{Funding}

This study was supported by funds from the Cancer Institute of New Jersey Cancer Prevention and Control pilot award (P30CA072720-19 to JJP) and National Cancer Institute (K07CA222158-01 to JJP). This study was also partly supported by the Columbia Population Research Center (P2CHD058486 to AGR), the Eunice Kennedy Shriver National Institute of Child Health \& Human Development of the National Institutes of Health (1R01HD087460-01 to AGR), the National Library of Medicine (1 K99LM012868 to SJM), and the Rutgers Center for Environmental Exposure and Disease (P30ES005022-30). This study's funding sponsors had no role in study design; collection, analysis, and interpretation of data; writing the report; nor the decision to submit the report.

\section{Availability of data and materials}

The datasets used and/or analysed during the current study are available from the corresponding author on reasonable request.

\section{Ethics approval and consent to participate}

This study was approved by the Institutional Review Board of Rutgers University (IRB study \#: Pro20170001267).

\section{Consent for publication \\ Not applicable.}

\section{Competing interests}

No financial disclosures were reported by the authors of this paper. The authors declare that they have no competing interests for publication.

\section{Author details}

${ }^{1}$ Department of Biostatistics and Epidemiology, Rutgers School of Public Health, Piscataway, NJ, USA. ${ }^{2}$ Rutgers Cancer Institute of New Jersey, New Brunswick, NJ, USA. ${ }^{3}$ Department of Clinical Analytics, SSM Health, St. Louis, MO, USA. ${ }^{4}$ Department of Epidemiology, Mailman School of Public Health, Columbia University, New York, USA. ${ }^{5}$ New Jersey Department of Health, New Jersey State Cancer Registry, Trenton, NJ, USA. ${ }^{6}$ Department of Epidemiology, University of Washington, Seattle, WA, USA.

Received: 4 January 2020 Accepted: 19 May 2020

Published online: 29 May 2020

\section{References}

1. Bancroft C, Joshi S, Rundle A, Hutson M, Chong C, Weiss CC, Genkinger J, Neckerman K, Lovasi G. Association of proximity and density of parks and objectively measured physical activity in the United States: a systematic review. Soc Sci Med. 2015;138:22-30.

2. McGrath $L$, Hopkins WG, Hinckson EA. Associations of objectively measured built-environment attributes with youth moderate-vigorous physical activity: a systematic review and meta-analysis. Sports Med. 2015;45(6):841-65.

3. Mayne SL, Jose A, Mo A, Vo L, Rachapalli S, Ali H, Davis J, Kershaw KN. Neighborhood disorder and obesity-related outcomes among women in Chicago. Int J Environ Res Public Health. 2018;15(7):1395.

4. Mooney SJ, DiMaggio CJ, Lovasi GS, Neckerman KM, Bader MDM, Teitler JO, Sheehan DM, Jack DW, Rundle AG. Use of Google street view to assess environmental contributions to pedestrian injury. Am J Public Health. 2016;106(3):462-9.

5. Branas CC, South E, Kondo MC, Hohl BC, Bourgois P, Wiebe DJ, MacDonald JM. Citywide cluster randomized trial to restore blighted vacant land and its effects on violence, crime, and fear. Proc Natl Acad Sci USA. 2018;115(12):2946-51.

6. Nguyen QC, Khanna S, Dwivedi P, Huang D, Huang Y, Tasdizen T, Brunisholz KD, Li F, Gorman W, Nguyen TT, et al. Using Google street view to examine associations between built environment characteristics and U.S. health outcomes. Prev Med Rep. 2019;14:100859.

7. Helbich M, Yao Y, Liu Y, Zhang JB, Liu PH, Wang RY. Using deep learning to examine street view green and blue spaces and their associations with geriatric depression in Beijing, China. Environ Int. 2019;126:107-17.

8. Fernandez-Nino JA, Bonilla-Tinoco LJ, Manrique-Espinoza BS, Salinas-Rodriguez A, Santos-Luna R, Roman-Perez S, Morales-Carmona E, Duncan DT. Neighborhood features and depression in Mexican older adults: a longitudinal analysis based on the study on global AGEing and adult health (SAGE), waves 1 and 2 (2009-2014). PLoS ONE. 2019;14(7):e0219540.

9. O'Brien DT, Farrell C, Welsh BC. Broken (windows) theory: a meta-analysis of the evidence for the pathways from neighborhood disorder to resident health outcomes and behaviors. Soc Sci Med. 2019;228:272-92.

10. Furr-Holden CDM, Lee MH, Milam AJ, Johnson RM, Lee KS, lalongo NS. The growth of neighborhood disorder and marijuana use among urban adolescents: a case for policy and environmental interventions. J Stud Alcohol Drugs. 2011;72(3):371-9.

11. Love your block (https://www.newarknj.gov/card/love-your-block). 
12. Zhang Z, Manjourides J, Cohen T, Hu Y, Jiang Q. Spatial measurement errors in the field of spatial epidemiology. Int J Health Geogr. 2016;15(1):21.

13. Day K, Boarnet M, Alfonzo M, Forsyth A. The Irvine-Minnesota inventory to measure built environments development. Am J Prev Med. 2006;30(2):144-52.

14. Bader MD, Mooney SJ, Lee YJ, Sheehan D, Neckerman KM, Rundle AG, Teitler JO. Development and deployment of the Computer Assisted Neighborhood Visual Assessment System (CANVAS) to measure healthrelated neighborhood conditions. Health Place. 2015;31:163-72.

15. Sampson RJ, Raudenbush SW, Earls F. Neighborhoods and violent crime: a multilevel study of collective efficacy. Science (New York, NY). 1997:277(5328):918-24

16. Sampson RJ, Raudenbush SW. Systematic social observation of public spaces: a new look at disorder in urban neighborhoods 1 . Am J Sociol. 1999;105(3):603-51.

17. Bethlehem JR, Mackenbach JD, Ben-Rebah M, Compernolle S, Glonti K, Bardos H, Rutter HR, Charreire H, Oppert JM, Brug J, et al. The SPOTLIGHT virtual audit tool: a valid and reliable tool to assess obesogenic characteristics of the built environment. Int J Health Geographics. 2014;13:52.

18. Badland HM, Opit S, Witten K, Kearns RA, Mavoa S. Can virtual streetscape audits reliably replace physical streetscape audits? J Urban Health Bull New York Acad Med. 2010;87(6):1007-16

19. Griew P, Hillsdon M, Foster C, Coombes E, Jones A, Wilkinson P. Developing and testing a street audit tool using Google Street View to measure environmental supportiveness for physical activity. Int J Behav Nutr Phys Activity. 2013;10:103.

20. Gullon P, Badland HM, Alfayate S, Bilal U, Escobar F, Cebrecos A, Diez J, Franco M. Assessing walking and cycling environments in the streets of Madrid: comparing on-field and virtual audits. J Urban Health Bull New York Acad Med. 2015;92(5):923-39.

21. Rzotkiewicz A, Pearson AL, Dougherty BV, Shortridge A, Wilson N. Systematic review of the use of Google Street View in health research: major themes, strengths, weaknesses and possibilities for future research. Health Place. 2018;52:240-6.

22. Curtis JW, Curtis A, Mapes J, Szell AB, Cinderich A. Using google street view for systematic observation of the built environment: analysis of spatio-temporal instability of imagery dates. Int J Health Geograph. 2013;12:53.

23. Clarke P, Ailshire J, Melendez R, Bader M, Morenoff J. Using Google Earth to conduct a neighborhood audit: reliability of a virtual audit instrument. Health Place. 2010;16(6):1224-9.

24. Kelly CM, Wilson JS, Baker EA, Miller DK, Schootman M. Using google street view to audit the built environment: inter-rater reliability results. Ann Behav Med. 2013;45:S108-12.

25. Mooney SJ, Bader MDM, Lovasi GS, Teitler JO, Koenen KC, Aiello AE, Galea S, Goldmann E, Sheehan DM, Rundle AG. Street audits to measure neighborhood disorder: virtual or in-person? Am J Epidemiol. 2017;186(3):265-73.

26. Wilson JS, Kelly CM, Schootman M, Baker EA, Banerjee A, Clennin M, Miller DK. Assessing the built environment using omnidirectional imagery. Am J Prev Med. 2012;42(2):193-9.

27. Mooney SJ, Bader MD, Lovasi GS, Neckerman KM, Teitler JO, Rundle AG. Validity of an ecometric neighborhood physical disorder measure constructed by virtual street audit. Am J Epidemiol. 2014;180(6):626-35.

28. Kruger DJ, ReischI TM, Gee GC. Neighborhood social conditions mediate the association between physical deterioration and mental health. Am J Community Psychol. 2007;40(3-4):261-71.

29. Hunter MCR, Brown DG. Spatial contagion: gardening along the street in residential neighborhoods. Landscape Urban Plan. 2012;105(4):407-16.

30. McClintock N, Mahmoudi D, Simpson M, Santos JP. Socio-spatial differentiation in the Sustainable City: a mixed-methods assessment of residential gardens in metropolitan Portland, Oregon, USA. Landscape Urban Plan. 2016:148:1-16.

31. Waller LA, Gotway CA. Applied spatial statistics for public health data. Hoboken: Wiley; 2004

32. Plascak JJ, Rundle AG, Babel RA, Llanos AA, LaBelle CM, Stroup AM, Mooney SJ. Drop-and-spin virtual neighborhood auditing: assessing built environment for linkage to health studies. Am J Prev Med. 2020;58(1):152-60.
33. Kepper MM, Sothern MS, Theall KP, Griffiths LA, Scribner RA, Tseng TS, Schaettle P, Cwik JM, Felker-Kantor E, Broyles ST. A reliable, feasible method to observe neighborhoods at high spatial resolution. Am J Prev Med. 2017;52(1):S20-30.

34. Mygind L, Bentsen P, Badland H, Edwards N, Hooper P, Villanueva K. Public open space desktop auditing tool-establishing appropriateness for use in Australian regional and urban settings. Urban Urban Gree. 2016;20:65-70.

35. Odgers CL, Caspi A, Bates CJ, Sampson RJ, Moffitt TE. Systematic social observation of children's neighborhoods using Google Street View: a reliable and cost-effective method. J Child Psychol Psychiatry. 2012;53(10):1009-17.

36. Silva V, Grande AJ, Rech CR, Peccin MS. Geoprocessing via google maps for assessing obesogenic built environments related to physical activity and chronic noncommunicable diseases: validity and reliability. J Healthc Eng. 2015;6(1):41-54

37. Vanwolleghem G, Ghekiere A, Cardon G, De Bourdeaudhuij I, D'Haese S, Geremia CM, Lenoir M, Sallis JF, Verhoeven H, Van Dyck D. Using an audit tool (MAPS Global) to assess the characteristics of the physical environment related to walking for transport in youth: reliability of Belgian data. Int J Health Geographics. 2016;15(1):41.

38. Pliakas T, Hawkesworth S, Silverwood RJ, Nanchahal K, Grundy C, Armstrong B, Casas JP, Morris RW, Wilkinson P, Lock K. Optimising measurement of health-related characteristics of the built environment: comparing data collected by foot-based street audits, virtual street audits and routine secondary data sources. Health Place. 2017:43:75-84.

39. Schootman M, Nelson EJ, Werner K, Shacham E, Elliott M, Ratnapradipa K, Lian M, McVay A. Emerging technologies to measure neighborhood conditions in public health: implications for interventions and next steps. Int J Health Geographics. 2016;15(1):20.

40. Gomez SL, Shariff-Marco S, DeRouen M, Keegan THM, Yen IH, Mujahid M, Satariano WA, Glaser SL. The impact of neighborhood social and built environment factors across the cancer continuum: current research, methodological considerations, and future directions. Cancer. 2015;121(14):2314-30.

41. Lynch SM, Rebbeck TR. Bridging the gap between biologic, individual, and macroenvironmental factors in cancer: a multilevel approach. Cancer Epidemiol Biomarkers Prev. 2013;22(4):485-95.

42. Martin DN, Lam TK, Brignole K, Ashing KT, Blot WJ, Burhansstipanov L, Chen JT, Dignan M, Gomez SL, Martinez ME. Recommendations for cancer epidemiologic research in understudied populations and implications for future needs. Cancer Epidemiol Biomark Prev. 2016;25(4):573-80.

43. Vanwolleghem G, Van Dyck D, Ducheyne F, De Bourdeaudhuij I, Cardon G. Assessing the environmental characteristics of cycling routes to school: a study on the reliability and validity of a Google Street View-based audit. Int J Health Geographics. 2014;13:19.

44. Hengl T, Heuvelink GBM, Rossiter DG. About regression-kriging: from equations to case studies. Comput Geosci UK. 2007:33(10):1301-15.

45. Lin YP, Cheng BY, Chu HJ, Chang TK, Yu HL. Assessing how heavy metal pollution and human activity are related by using logistic regression and kriging methods. Geoderma. 2011;163(3-4):275-82.

46. Southworth M. Walkable suburbs?: an evaluation of neotraditional communities at the urban edge. J Am Plan Assoc. 1997;63(1):28-44.

47. Hayden D. Building suburbia: green fields and urban growth, 1820-2000. New York: Vintage Books; 2004.

48. Maghelal PK, Capp CJ. Walkability: a review of existing pedestrian indices. J Urban Reg Inform Syst Assoc. 2011;23(2):5.

49. Bureau USC: 2010 Census of population and housing, summary file 1 2010. U.S. Census Bureau; vol 2015; Washington, DC. 2011.

50. Earls FJ, Brooks-Gunn J, Raudenbush SW, Sampson RJ: Project on human development in Chicago neighborhoods: community survey, 1994-1995. In.: Inter-university Consortium for Political and Social Research (ICPSR) distributor; 2007.

51. Clifton KJ, Smith ADL, Rodriguez D. The development and testing of an audit for the pedestrian environment. Landscape Urban Plan. 2007;80(1-2):95-110.

52. Diggle P: Statistical analysis of spatial point patterns, vol 2. London, New York: Arnold; Distributed by Oxford University Press; 2003.

53. Baddeley A, Rubak E, Turner R. Spatial point patterns methodology and applications with R. Boca Raton: CRC Press; 2015.

54. Journel AG, Huijbregts CJ. Mining geostatistics. London; New York: Academic Press; 1978. 
55. Araki S, Yamamoto K, Kondo A. Application of regression kriging to air pollutant concentrations in japan with high spatial resolution. Aerosol Air Qual Res. 2015;15(1):234-41.

56. Bourennane $H$, King D, Couturier A. Comparison of kriging with external drift and simple linear regression for predicting soil horizon thickness with different sample densities. Geoderma. 2000;97(3-4):255-71.

57. Hosmer DW, Lemeshow S. Applied logistic regression, vol. 2. New York: Wiley; 2000

58. Manson S, Schroeder J, Riper DV, Ruggles S: IPUMS National Historical Geographic Information System: Version 14.0 (Database), vol 2019. Minneapolis, MN: IPUMS; 2019.

59. Environmental systems research I: ArcGIS desktop. Version 10.5. Redlands, CA: Environmental Systems Research Institute 2020.

60. Sas I: SAS/STAT 9.4 user's guide: SAS Institute. 2014.

61. Baddeley A, Turner R: Package 'spatstat'. 2015.

62. Duncan DT, Aldstadt J, Whalen J, Melly SJ, Gortmaker SL. Validation of walk score (r) for estimating neighborhood walkability: an analysis of Four US metropolitan areas. Int J Environ Res Public Health. 2011;8(11):4160-79.

63. Marco M, Gracia E, Martin-Fernandez M, Lopez-Quilez A. Validation of a google street view-based neighborhood disorder observational scale. J Urban Health Bull New York Acad Med. 2017;94(2):190-8.

64. Remigio RV, Zulaika G, Rabello RS, Bryan J, Sheehan DM, Galea S, Carvalho MS, Rundle A, Lovasi GS. A local view of informal urban environments: a mobile phone-based neighborhood audit of street-level factors in a brazilian informal community. J Urban Health. 2019;96(4):537-48.

65. Cressie NAC. Statistics for spatial data, vol. Rev. New York: Wiley; 1993.

66. Orfield G, Ee J, Frankenberg E, Siegel-Hawley G. "Brown" at 62: school segregation by race, poverty and state. Los Angeles: Civil Rights Project; 2016

67. Acevedo-Garcia D. Zip code-level risk factors for tuberculosis: neighborhood environment and residential segregation in New Jersey, 1985-1992. Am J Public Health. 2001;91(5):734-41.
68. Chaix B, Kestens Y, Perchoux C, Karusisi N, Merlo J, Labadi K. An interactive mapping tool to assess individual mobility patterns in neighborhood studies. Am J Prev Med. 2012:43(4):440-50.

69. Sampson RJ, Sharkey P. Neighborhood selection and the social reproduction of concentrated racial inequality. Demography. 2008;45(1):1-29.

70. Oakes JM, Andrade KE, Biyoow IM, Cowan LT. Twenty years of neighborhood effect research: an assessment. Curr Epidemiol Rep. 2015;2(1):80-7.

71. Jokela M. Are neighborhood health associations causal? a 10-year prospective cohort study with repeated measurements. Am J Epidemiol. 2014;180(8):776-84

72. Nuckols J, Airola M, Colt J, Johnson A, Schwenn M, Waddell R, Karagas M, Silverman $\mathrm{D}$, Ward $\mathrm{MH}$. The impact of residential mobility on exposure assessment in cancer epidemiology. Epidemiology. 2009;20(6):S259-60.

73. Deziel NC, Ward MH, Bell EM, Whitehead TP, Gunier RB, Friesen MC, Nuckols JR. Temporal variability of pesticide concentrations in homes and implications for attenuation bias in epidemiologic studies. Environ Health Perspect (Online). 2013;121(5):565

74. Salesses $P$, Schechtner $K$, Hidalgo CA. The collaborative image of the city: mapping the inequality of urban perception. Plos One. 2013;8(7):e68400.

75. Sources of photography (https://www.google.com/streetview/explore/).

76. Cressie N, Wikle CK. Statistics for spatio-temporal data. New Jersey: Wiley; 2015.

77. Wang RY, Liu Y, Lu Y, Yuan Y, Zhang JB, Liu PH, Yao Y. The linkage between the perception of neighbourhood and physical activity in Guangzhou, China: using street view imagery with deep learning techniques. Int J Health Geographics. 2019;18:18.

\section{Publisher's Note}

Springer Nature remains neutral with regard to jurisdictional claims in published maps and institutional affiliations.
Ready to submit your research? Choose BMC and benefit from:

- fast, convenient online submission

- thorough peer review by experienced researchers in your field

- rapid publication on acceptance

- support for research data, including large and complex data types

- gold Open Access which fosters wider collaboration and increased citations

- maximum visibility for your research: over $100 \mathrm{M}$ website views per year

At BMC, research is always in progress.

Learn more biomedcentral.com/submissions 